\title{
Learning to Rank for Uplift Modeling
}

\begin{tabular}{|r|l|}
\hline Journal: & Transactions on Knowledge and Data Engineering \\
\hline Manuscript ID & TKDE-2020-04-0372.R1 \\
\hline Manuscript Type: & Regular \\
\hline Keywords: & $\begin{array}{l}\text { Learning to rank, Uplift modeling, Causal classification, Performance } \\
\text { measures }\end{array}$ \\
\hline
\end{tabular}

\section{SCHOLARONE \\ Manuscripts}




\title{
Learning to Rank for Uplift Modeling
}

\author{
Floris Devriendt, Jente Van Belle, Tias Guns, and Wouter Verbeke
}

\begin{abstract}
Causal classification concerns the estimation of the net effect of a treatment on an outcome of interest at the instance level, i.e., of the individual treatment effect (ITE). For binary treatment and outcome variables, causal classification models produce ITE estimates that essentially allow one to rank instances from a large positive effect to a large negative effect. Often, as in uplift modeling (UM), one is merely interested in this ranking, rather than in the ITE estimates themselves. In this regard, we investigate the potential of learning to rank (L2R) techniques to learn a ranking of the instances directly. We propose a unified formalization of different binary causal classification performance measures from the UM literature and explore how these can be integrated into the L2R framework. Additionally, we introduce a new metric for UM with L2R called the promoted cumulative gain (PCG). We employ the L2R technique LambdaMART to optimize the ranking according to PCG and show improved results over the use of standard L2R metrics and equal to improved results when compared with state-of-the-art UM. Finally, we show how L2R techniques can be used to specifically optimize for the top- $k$ fraction of the ranking in a UM context, however, these results do not generalize to the test set.
\end{abstract}

\section{INTRODUCTION}

C AUSAL classification models estimate for each instance the causal effect of a treatment on an outcome variable of interest, i.e., the individual treatment effect (ITE) [1]. This causal inference task is encountered in the literature under various names, e.g., heterogeneous treatment effect estimation [2], individualized treatment rule learning [3], conditional average treatment effect estimation [4], and uplift modeling [5]. Causal classification models have been applied in various domains to maximize the effectiveness of e.g., personalized medicine [6] and marketing campaigns [7]. Another application is determining which customers to target with a retention campaign to maximize reduction in churn while also minimizing the use of resources [8].

In this work, we focus on binary causal classification in that we consider both treatment and outcome to be binary variables. In this setting, ITE estimates allow one to rank instances from a large positive effect to a large negative effect. Often, one is merely interested in this ranking rather than in the ITE estimates themselves. This specific ranking objective is typically encountered in the uplift modeling (UM) literature [5]. Learning to rank (L2R) techniques, which stem from the information retrieval community, comprise techniques specifically designed to optimize the quality of predicted rankings directly, rather than the quality of predicted values that serve to rank instances [9]. This work investigates whether $\mathrm{L} 2 \mathrm{R}$ techniques can be successfully used in the context of UM.

While existing UM techniques are in fact already approaches to L2R since standard classification techniques can be considered as 'pointwise' approaches to L2R (see Section 3 ), we present different ways to formulate $\mathrm{UM}$ as an $\mathrm{L} 2 \mathrm{R}$ problem. As this is directly linked to the different evaluation

- F. Devriendt, J. Van Belle, and T. Guns are with the Data Analytics Laboratory, Solvay Business School, Vrije Universiteit Brussel, Brussels, Belgium.

- W. Verbeke is with the Faculty of Economics and Business, Katholieke Universiteit Leuven, Leuven, Belgium.

Correspondence e-mail: wouter.verbeke@kuleuven.be

Manuscript received $x x x x, x x x x$; revised $x x x x, x x x x$. measures proposed to assess the quality of produced rankings in UM, we first provide an overview of these measures. We then consider how suited existing L2R measures are for UM and introduce a new L2R metric for this purpose, called the promoted cumulative gain, by translating the uplift metric Area Under the Uplift Curve to an L2R measure which can be directly used together with the LambdaMART L2R technique.

Finally, in both UM and information retrieval, often only the top- $k$ fraction of the ranking is of interest to the user [5]. However, while L2R comprises techniques and measures designed to optimize for the top- $k$ specifically, current UM techniques aim to optimize the entire ranking. Therefore, we will investigate whether specifically optimizing for the top$k$ by using L2R techniques can be successfully applied to $\mathrm{UM}$. To the best of our knowledge, optimizing for the top- $k$ has not been investigated before in UM.

Our contributions are:

- We investigate and experimentally compare the main UM evaluation measures in use today, and propose a unified formalization in which all measures can be unambiguously written.

- We explore the different ways in which UM can be formulated as an L2R problem.

- We introduce a new metric for UM with L2R, called the promoted cumulative gain.

- We empirically evaluate the different L2R formulations for UM on multiple datasets.

- We investigate optimizing specifically for the top- $k$ in UM by using L2R techniques, though the benefit of top- $k$ learning is shown to be limited as the results do not generalize to the test set.

- We compare different state-of-the-art UM techniques with our best performing L2R formulation and show that $\mathrm{L} 2 \mathrm{R}$ is a viable alternative to the existing $\mathrm{UM}$ methodology.

The rest of the paper is organized as follows: in Section 2 we formally introduce causal classification and UM, discuss related work and elaborate on the evaluation measures used 
in the UM literature. Section 3 covers L2R and its measures, how UM can be formulated as an L2R problem and introduces a new metric for the L2R framework. In Section 4, we describe the experiments and report the results. Section 5 discusses the results and finally Section 6 presents our conclusions.

\section{Causal Classification \& Uplift Modeling}

Causal classification is about estimating the class of an instance in function of the treatment that is applied. Hence, it is equivalent to estimating the ITE, i.e., the causal effect of a treatment on an outcome of interest at the instance level [1]. Therefore, causal classification models can be used to optimize treatment assignment to instances with the aim to maximize (or, depending on the application at hand, to minimize) the outcome of interest. In this work, we focus on binary causal classification in that we consider both treatment and outcome to be binary variables.

Suppose that we have a dataset $\mathcal{D}=\{(x, y, t)\}$ of $n$ instances with each a feature vector $x \in \mathcal{X}$, response variable $y \in\{0,1\}$ and treatment indicator $t \in\{0,1\}$. Following the Neyman-Rubin potential outcomes framework [10], the ITE can be formally defined as:

$$
\tau(x)=\mathbb{E}\left[Y^{(1)}-Y^{(0)} \mid x\right]
$$

where $Y^{(1)}$ and $Y^{(0)}$ are the two potential outcomes and correspond to the response $y$ of an instance belonging to the treatment $(t=1)$ and control $(t=0)$ group, respectively. Note that it is generally assumed that some instances respond $(y=1)$ without being treated $(t=0)$ (e.g., natural healing or subscribing to a product independent of advertising). The main difficulty in ITE estimation is that $\tau(x)$ is not directly observable since we can only observe one of the potential outcomes for a particular instance. That is, we can observe the outcome after treating or not treating, but can not know what the outcome would have been for the opposite treatment choice. This is commonly known as The Fundamental Problem Of Causal Inference [11].

In this work, we focus on estimating the ITE in randomized controlled trial (RCT) settings. In such setting, the treatment was administered randomly and independent of $x$ and $\tau(x)$ can be estimated as [4], [12]:

$$
\hat{\tau}(x)=P(y=1 \mid x, t=1)-P(y=1 \mid x, t=0) .
$$

The ITE is thus defined as the difference between the probability of an instance to respond if treated minus the probability of an instance to respond if not treated.

As mentioned in the introduction, in case of binary treatment and outcome variables, ITE estimates allow one to rank instances from a large positive effect to a large negative effect. In many cases, one is merely interested in this ranking rather than in the ITE estimates themselves. This specific setting, which is the focus of this work, lies at the core of the UM literature [5].

UM has as goal to rank an unseen set of instances, e.g. customers, by their estimated $\hat{\tau}(x)$ and to target a highly ranked fraction of this set of instances. For example, in marketing or churn prediction, the size of the fraction is determined by the campaign budget, e.g., the top 1000 or 10000 customers. Given a limited budget, these customers are expected to be most likely to respond when targeted with the campaign.

\subsection{Related Work}

UM techniques can be grouped into data preprocessing and data processing approaches. In the data preprocessing approaches, after pre- or postprocessing data and outcomes, existing out-of-the-box learning methods are used. In the data processing approaches, new learning methods and methodologies are developed that aim to estimate $\tau(x)$ more directly. For an in depth discussion on UM techniques we refer to [5], [13].

A popular and generic data preprocessing approach is the flipped label approach, also called the class transformation approach [14], [15]. In this approach, a new target variable $z \in\{0,1\}$ is created where $z=1$ if either: $t=1$ and $y=1$ or $t=0$ and $y=0$; and $z=0$ otherwise. Due to this class transformation, estimation of $\tau(x)$ is converted into a binary classification problem with label $z$, allowing us to adopt any standard classification technique [15].

Other data preprocessing approaches extend the set of features to allow for the estimation of $\tau(x)$. An example is grouping together the instances from both treatment and control group and including a dummy variable that denotes to which group an instance belongs. A model is then developed from: (1) the original features; (2) the added dummy variable; and (3) interaction variables between the features and the dummy variable [16], [17]. This model can then be used to estimate two response probabilities for a new instance, once as if the instance belongs to the treatment group and once as if it belongs to the control group. Subtracting the probabilities returns $\hat{\tau}(x)$.

Data processing approaches comprise both indirect and direct estimation approaches. Indirect estimation approaches include the two model approach. This approach builds two separate models to predict the response probabilities, one for the treatment group and one for the control group. For a new instance, the probability of responding is estimated with each model. Afterwards, the probabilities are subtracted to obtain $\hat{\tau}(x)$.

Direct estimation approaches are typically adaptations of decision tree algorithms such as CART [18] or CHAID [19]. Proposed adaptations include modified splitting criteria and dedicated pruning techniques. Examples of tree-based techniques include significance-based uplift trees [20], decision trees with information theory-inspired splitting criteria [21] and uplift random forest and causal conditional inference trees [22]. However, there is also a group of direct estimation techniques that builds on support vector machines [23], [24], [25].

Finally, note that the overview above focuses on techniques to estimate ITE in RCT settings. However, ITE estimation based on observational data with treatment selection bias is also an active research area in the field of causal machine learning. For recent works on this subject, one may refer to [1], [26] and references therein.

Our work differs from all the above in that we focus on the link between UM and L2R, both when treatment and control groups are handled as separate subsets or as one joint set. 


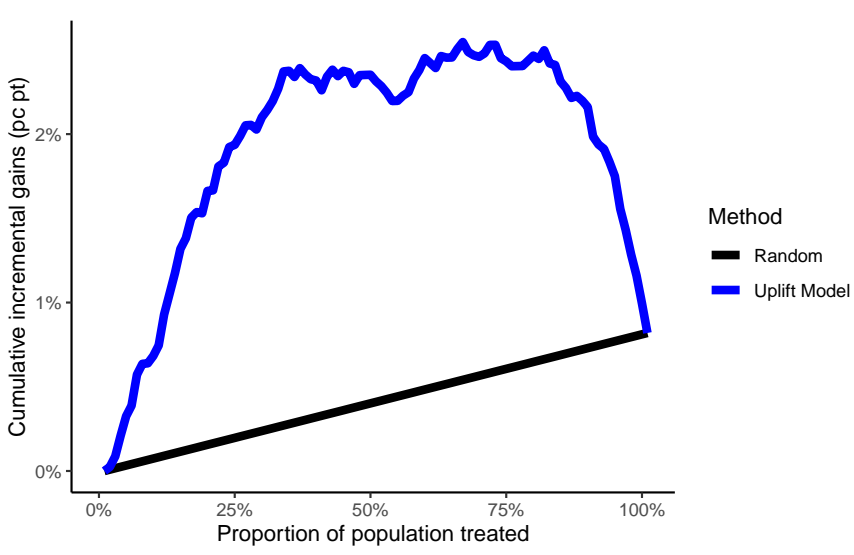

Fig. 1: A cumulative incremental gains curve (blue) and the expected gains from random treatments (black).

\subsection{Performance Measures for Evaluating Uplift Models}

Because $\tau(x)$ is unobservable, we can not directly measure the quality of the estimated $\hat{\tau}(x)$ values. However, since in UM one is rather interested in the ranking that results from ranking instances by $\hat{\tau}(x)$, the norm is to evaluate the quality of this ranking. [20] propose to do this by computing and plotting the cumulative incremental gains for an incrementally larger subgroup of the ranked population, i.e., the lift in response rate as a result of treating more instances or the cumulative incremental treatment effect.

Figure 1 shows an example, where the blue line represents the cumulative incremental gains as a function of the selected fraction of the ranked population. The black line represents the expected cumulative incremental gains for a random subsample of that size, called the random baseline. A good uplift model ranks instances likely to respond when treated higher, leading to higher estimated cumulative incremental gains in the early parts of the plot.

Interestingly, many different ways for computing and visualizing the cumulative incremental gains have been proposed in the literature. Two variants exist with a different name: the Qini Curve and the Uplift Curve. In this work, we focus on the difference of how the cumulative incremental gain values are computed for both variants. However, note that also other differences between the curves occur in the literature, e.g., what values are plotted, and how a single measure such as Area Under the Curve is derived.

Even for the Qini and Uplift variants, there are no unique definitions. We analyzed the literature, and identified two main differences. The first one relates to whether the ranking is computed for each group (i.e., treatment and control group) separately or for one joint group. If the ranking is computed separately, the top 10\% instances are the top 10\% instances of the treatment group and the top $10 \%$ instances of the control group. However, if the ranking is computed jointly, the top $10 \%$ instances originate from both groups. Hence, in the joint setup, the proportions of instances of each group represented in the ranking can differ from the global proportions of treatment and control groups. The second difference is linked to the potential imbalance of the treatment and control groups as it relates to whether the cumulative incremental gains are computed in (rebalanced) absolute numbers of instances or in relative terms.

An overview of the different definitions occurring in the literature, structured according to the identified differences discussed above, is provided in Table 2. Before discussing differences between the definitions in more detail, we first introduce notation allowing us to formalize the different definitions.

For evaluation, we assume the presence of a predictive model $\hat{u}$ that allows us to rank a dataset $\mathcal{D}$ in decreasing order, i.e., instances are ranked from high to low $\hat{\tau}(x)$ (see Section 2.1). Note that $\hat{u}$ does not necessarily estimates $\hat{\tau}(x)$ based on the probabilities as in Equation 2 directly. We denote the total number of treated $(t=1)$ and control $(t=0)$ instances among the top- $k$ ranked instances by $N^{\mathcal{T}}(\mathcal{D}, k)$ and $N^{\mathcal{C}}(\mathcal{D}, k)$, and the number of treated and control responders $(y=1)$ among the top- $k$ ranked instances by $R^{\mathcal{T}}(\mathcal{D}, k)$ and $R^{\mathcal{C}}(\mathcal{D}, k)$, respectively.

In the literature, the lift in response rate is often computed and compared for both the treatment and control group separately. To formalize this, we introduce the subsets $\mathcal{T}$ and $\mathcal{C}$, with $\mathcal{T}$ the subset of treated instances and $\mathcal{C}$ the subset of instances that belong to the control group. In line with the notations introduced in the previous paragraph for rankings covering both the treatment and control group, we denote the number of treated and control responders among the top- $k$ ranked instances per group by $R(\mathcal{T}, k)$ and $R(\mathcal{C}, k)$.

To clarify the notation introduced, consider Table 1 which shows a minimal example of a ranked dataset. For the number of treated instances among the top 1 and top 3 instances in the dataset, one would write $N^{\mathcal{T}}(\mathcal{D}, 1)=1$ and $N^{\mathcal{T}}(\mathcal{D}, 3)=2$, respectively. Likewise, to obtain the number of treated responders among the top 1 and top 3 instances in the dataset, one would write $R^{\mathcal{T}}(\mathcal{D}, 1)=0$ and $R^{\mathcal{T}}(\mathcal{D}, 3)=1$, respectively. The above notations relate to the joint scenario, in which a ranking is computed for the treatment and control instances as one group. In the separate scenario, on the other hand, both the treatment and control group have their own ranking. Therefore, to obtain the number of treated responders among the top 1 and top 3 instances, one would write $R(\mathcal{T}, 1)=0$ and $R(\mathcal{T}, 3)=2$, respectively. Notice the difference in the numbers obtained for the top 3 case.

TABLE 1: Minimal example of dataset ranked in decreasing order to demonstrate differences in notations.

\begin{tabular}{|c|c|c|}
\hline & $y$ & $t$ \\
\hline$i_{1}$ & 0 & 1 \\
\hline$i_{2}$ & 1 & 1 \\
\hline$i_{3}$ & 1 & 0 \\
\hline$i_{4}$ & 1 & 1 \\
\hline
\end{tabular}

Table 2 shows the different evaluation measures for UM proposed in the literature. For the separate scenarios, we use $p$ to denote a percentage, where $p|\mathcal{T}|$ and $p|\mathcal{C}|$ are then the corresponding absolute numbers of instances being considered from the treatment and control groups, respectively. The value function $V()$ returns the cumulative incremental gains value for the first $p$-percent of the population, or for all instances up to and including instance $k$. 
TABLE 2: Evaluation measures for UM. Two main approaches are considered, the Qini Curve and the Uplift Curve, both over two dimensions: ranking the data separately per group or jointly over all data, and expressing the cumulative incremental gains in absolute or relative terms.

\begin{tabular}{|c|c|c|c|}
\hline Rank & Count & Qini Curve & Uplift Curve \\
\hline \multirow[t]{2}{*}{ Sep. } & Abs. & $\begin{array}{c}V(p)=R(\mathcal{T}, p|\mathcal{T}|)-R(\mathcal{C}, p|\mathcal{C}|) \frac{|\mathcal{T}|}{|\mathcal{C}|} \\
\text { [12], [20], [27], [28] }\end{array}$ & $\begin{array}{c}V(p)=R(\mathcal{T}, p|\mathcal{T}|)-R(\mathcal{C}, p|\mathcal{C}|) \\
{[24]}\end{array}$ \\
\hline & Rel. & / & $\begin{array}{c}V(p)=\frac{R(\mathcal{T}, p|\mathcal{T}|)}{|\mathcal{T}|}-\frac{R(\mathcal{C}, p|\mathcal{C}|)}{|\mathcal{C}|} \\
{[15],[21],[25],[29],[30],[31],[32],[33]}\end{array}$ \\
\hline \multirow[t]{2}{*}{ Joint } & Abs. & $\begin{array}{c}V(k)=R^{\mathcal{T}}(\mathcal{D}, k)-R^{\mathcal{C}}(\mathcal{D}, k) \frac{N^{\mathcal{T}}(\mathcal{D}, k)}{N^{\mathcal{C}}(\mathcal{D}, k)} \\
\text { [27], [34] }\end{array}$ & $V(k)=\left(\frac{R^{\mathcal{T}}(\mathcal{D}, k)}{N^{\mathcal{T}}(\mathcal{D}, k)}-\frac{R^{\mathcal{C}}(\mathcal{D}, k)}{N^{\mathcal{C}}(\mathcal{D}, k)}\right) *\left(N^{\mathcal{T}}(\mathcal{D}, k)+N^{\mathcal{C}}(\mathcal{D}, k)\right)$ \\
\hline & Rel. & \multicolumn{2}{|c|}{$\begin{aligned} V(k) & =\frac{R^{\mathcal{T}}(\mathcal{D}, k)}{|\mathcal{T}|}-\frac{R^{\mathcal{C}}(\mathcal{D}, k)}{|\mathcal{C}|} \\
{[12],[36] } & \end{aligned}$} \\
\hline
\end{tabular}

The first curve that occurs in the literature is the Qini Curve [28]. This curve plots the absolute number of incremental responses of the treated group compared to as when there is no treatment. To obtain a balanced comparison, however, the number of responders among the top $p$-percent of the control group is adjusted to neutralize the effect of different treatment and control group sizes. The values for the Qini Curve are then obtained by:

$$
V(p)=R(\mathcal{T}, p|\mathcal{T}|)-R(\mathcal{C}, p|\mathcal{C}|) \frac{|\mathcal{T}|}{|\mathcal{C}|} .
$$

The Qini Curve has since been used and modified by several researchers to evaluate the performance of uplift models.

An alternative to the Qini Curve is the Uplift Curve. The Uplift Curve is obtained by subtracting two separate lift curves, one for the treatment and one for the control group, using the same model [21]. In [30] the authors measure the cumulative incremental gains by subtracting the gain obtained from the first $p$-percent of ranked instances of the control group from the gain obtained from the first $p$-percent of ranked instances of the treatment group. A normalization factor, i.e., dividing by the respective group sizes, is added to account for overall imbalance in treatment and control groups. The corresponding formula is then:

$$
V(p)=\frac{R(\mathcal{T}, p|\mathcal{T}|)}{|\mathcal{T}|}-\frac{R(\mathcal{C}, p|\mathcal{C}|)}{|\mathcal{C}|} .
$$

Note that from a modeling point of view, the above equation is equivalent to Equation 3 as it can be obtained by dividing Equation 3 by the constant $|\mathcal{T}|$. Hence, both measures will give rise to the same conclusions when used for comparing different models.

In [24], the authors follow the same reasoning that was used to obtain Equation 4, however, the difference is that they measure the cumulative incremental gains in absolute terms. The authors do not mention any normalization applied in calculating the cumulative incremental gains:

$$
V(p)=R(\mathcal{T}, p|\mathcal{T}|)-R(\mathcal{C}, p|\mathcal{C}|) .
$$

All above definitions of Qini and Uplift curves evaluate uplift models in a separate manner, i.e., the top p-percent of the treatment group is compared with the top p-percent of the control group. A different approach is to consider both the treatment and control groups as one joint group and evaluate targeting the top- $k$ from that group, which is closer to how uplift models are to be used in practice.
In the joint relative setup, there is no clear distinction between the Qini and Uplift curves, as they are both obtained as follows [12], [36]:

$$
V(k)=\frac{R^{\mathcal{T}}(\mathcal{D}, k)}{|\mathcal{T}|}-\frac{R^{\mathcal{C}}(\mathcal{D}, k)}{|\mathcal{C}|} .
$$

For the joint absolute setting, however, both Qini and Uplift variants can be distinguished. In the first variant, the cumulative incremental gains are expressed in rebalanced absolute numbers [34]:

$$
V(k)=R^{\mathcal{T}}(\mathcal{D}, k)-R^{\mathcal{C}}(\mathcal{D}, k) \frac{N^{\mathcal{T}}(\mathcal{D}, k)}{N^{\mathcal{C}}(\mathcal{D}, k)} .
$$

Note that this measure rebalances the responder counts based on the numbers of treated and control instances among the top- $k$ ranked instances, instead of the overall imbalance $|\mathcal{T}| /|\mathcal{C}|$. The second variant is proposed by [35] and uses a different approach to obtain the cumulative incremental gains in rebalanced absolute numbers:

$V(k)=\left(\frac{R^{\mathcal{T}}(\mathcal{D}, k)}{N^{\mathcal{T}}(\mathcal{D}, k)}-\frac{R^{\mathcal{C}}(\mathcal{D}, k)}{N^{\mathcal{C}}(\mathcal{D}, k)}\right) *\left(N^{\mathcal{T}}(\mathcal{D}, k)+N^{\mathcal{C}}(\mathcal{D}, k)\right)$.

Here, the responder counts are divided by their respective number of instances among the top- $k$ ranked instances before subtraction, of which the result is then multiplied by the total number of instances considered in the ranking.

In addition to constructing a Qini or Uplift Curve according to one of the definitions above, we can also calculate the Area Under the Qini or Uplift Curve, hereinafter referred to as AUUC, to obtain a single numerical quantity to measure and compare the performance among uplift models. Sometimes the quantity obtained is reduced by the AUUC of the baseline [12], [28], however, in this paper we ignore this constant.

For any of the joint setting definitions from Table 2, the AUUC is defined as:

$$
A U U C=\int_{0}^{1} V(x) d x=\sum_{k=1}^{n} V(k) .
$$

For the separate setting definitions, we make the following approximation over 100 intervals:

$$
A U U C=\int_{0}^{1} V(x) d x \approx \sum_{p^{\prime}=1}^{100} V\left(p=\frac{p^{\prime}}{100}\right) .
$$


Note that in the literature, it is common to use only 10 groups (or deciles), though this does not provide much granularity.

The different evaluation measures are empirically compared in Section 4.2. However, as our goal is not only to evaluate rankings but also to optimize rankings, we turn to $\mathrm{L} 2 \mathrm{R}$ in the next section.

\section{Learning to Rank (L2R)}

L2R finds its origin in the Information Retrieval (IR) domain. IR is defined as finding material (usually documents) of an unstructured nature (usually text) that satisfies an information need from within large collections (usually stored on computers) [37]. Ranking is a core problem in IR, as it is an important part of many IR problems (e.g., document retrieval, collaborative filtering and product rating) [9]. In what follows, we use document retrieval as example to illustrate how L2R works and to establish the connection between L2R and UM.

A search engine is the most common example of a document retrieval system. The web consists of an extremely large amount of documents (i.e., webpages) and finding relevant documents is a difficult task. A search engine has multiple components, but one of its most crucial ones is the ranker. The ranker is responsible for matching the request of the user (i.e., the query) with relevant indexed documents. The goal of a ranking algorithm is to produce a ranked list of documents according to its relevance to a given query [9].

L2R algorithms can essentially be categorized into three groups: pointwise, pairwise and listwise approaches [9]. The pointwise approach predicts the relevance of each document and uses these final scores to rank all documents considered. Standard classification techniques can be considered as pointwise approaches to $\mathrm{L} 2 \mathrm{R}$ as they can be used to discriminate between 'relevant' and 'not relevant' documents. One limitation of a pointwise approach is that the interdependency between documents is not taken into consideration, meaning that the loss function used does not consider the documents' final place in the ranking. The pairwise approach takes as input pairs of documents and outputs for each pair which of the two documents is preferred in terms of relevance by relying on a classification model. For a entire list of documents, one thus obtains as output relative orderings for pairs of documents. However, deriving the position of all documents in the final ranking from this output is a difficult problem. Finally, the listwise approach takes as input the entire list of documents and directly outputs a ranking of all documents. This approach is closest to the L2R ideology as there is no mismatch between the learning stage and the final output of the algorithm (as is the case for pointwise and pairwise approaches).

One of the most well-known and versatile techniques in L2R is LambdaMART [38], [39]. LambdaMART combines two methods previously proposed in the field, namely LambdaRank and Multiple Additive Regression Trees (MART). A core idea of LambdaRank, which is itself an extension of RankNet, is that it can directly optimize a ranking measure, even if it is non-differentiable, by relying on so-called $\lambda$-gradients. These gradients are determined heuristically by multiplying the gradients of a pairwise loss function by the difference obtained in the ranking metric under consideration due to swapping the pair's positions in the ranking. Combining LambdaRank gradients with the learning algorithm Multiple Additive Regression Trees (MART), which is a gradient boosted tree algorithm, gives us LambdaMART. For more information, one may refer to [38], [39].

Whilst a pairwise loss function is used in obtaining $\lambda$ gradients, the second factor depends on the global structure of the entire list of documents. Therefore, LambdaMART can be considered a listwise approach. Next to research on nonparametric methods such as LambdaMART, there is also research on parametric (model-based) listwise L2R methods, such as ListNet [40] and Plackett-Luce ranking models [41]. However, as the general objective of this work is to explore the potential of L2R for UM, in what follows we focus on adopting the LambdaMART L2R technique for UM. A broader exploration of L2R methods for UM is identified as a prime topic for further research.

\subsection{Performance Measures for Evaluating L2R Models}

Commonly used performance metrics in L2R are [42], [43], [44]: Precision (P), Mean Average Precision (MAP), Cumulative Gain (CG) and (Normalized) Discounted Cumulative Gain (DCG). Different metrics are used depending on whether relevance values are binary or graded, e.g., graded according to a five-star rating system. Below, we briefly discuss each of the above measures.

\subsubsection{Binary Relevance}

When working with binary relevance values, an instance $i$ (i.e., a document in document retrieval) is either 'relevant' or 'not relevant', i.e., rel $_{i} \in\{0,1\}$.

The Precision at $k(P(k))$ corresponds to the proportion of relevant instances $\left(\right.$ rel $\left._{i}=1\right)$ among the top- $k$ ranked instances:

$$
P(k)=\frac{\sum_{i=1}^{k} r e l_{i}}{k} .
$$

The Average Precision of a query $q$, consisting of $|q|$ instances, sums over all instances in the query and computes the average of the $P(k)$ values at every $k$ where a relevant instance is positioned:

$$
\operatorname{Avg} P(q)=\frac{\sum_{i=1}^{|q|} P(i)}{\sum_{i=1}^{|q|} \text { rel }_{i}} .
$$

The Mean Average Precision (MAP) is the mean of Average Precisions over a set of $Q$ queries:

$$
M A P(Q)=\frac{\sum_{q \in Q} \operatorname{Avg} P(q)}{|Q|} .
$$

\subsubsection{Graded Relevance}

Here, the relevance is assumed to be given as a graded score, e.g., $\mathrm{rel}_{i} \in\{1,2, \ldots, 5\}$, where a higher value means higher relevancy. Evaluation metrics can be modified accordingly, as highly relevant instances are more valuable than marginally relevant instances, which in turn are more valuable than not relevant instances. 
The Cumulative Gain (CG) of a ranked list is the sum of all relevance values of the ranked list of a single query $q$, up to point $k$ :

$$
C G(k)=\sum_{i=1}^{k} r_{e} l_{i}
$$

A limitation of CG is that it does not consider the connection between ranking position and instance relevancy [42].

An alternative that takes this connection into account, i.e., that considers whether the instances with highest relevancy do appear at the top of the ranking, is the Discounted Cumulative Gain (DCG). To achieve this, graded relevance values are discounted logarithmically proportional to the ranking positions [42]:

$$
D C G(k)=\sum_{i=1}^{k} \frac{\text { rel }_{i}}{\log _{2}(i+1)} .
$$

An alternative formulation for DCG does exist, which places more emphasis on the relevance values by using $2^{\text {rel }_{i}}-1$ instead of $\mathrm{rel}_{i}$ in the numerator [45]. When relevance values are binary, both formulations are equal [46].

Different queries can relate to different numbers of instances. To fairly compare a rankers' performance among multiple queries of different sizes, one can normalize the DCG of each query to obtain scores in the range of $[0,1]$. The Normalized Discounted Cumulative Gain (NDCG) metric normalizes DCG by dividing the achieved DCG of each query by its Ideal Discounted Cumulative Gain (IDCG), where the IDCG is obtained by sorting all instances according to their relevance values resulting in the maximum possible DCG [42]:

$$
\begin{aligned}
\operatorname{IDCG}(k) & =\sum_{i=1}^{k} \frac{r e l_{i}^{\prime}}{\log _{2}(i+1)} \\
N D C G(k) & =\frac{D C G(k)}{I D C G(k)}
\end{aligned}
$$

where $r e l_{i}^{\prime}$ is the best possible relevance value for instance $i$ as it results from the best possible ranking. By averaging over a set of queries $Q$, one can obtain the mean DCG and NDCG (similar to Equation 13).

\subsection{Uplift Modeling as Learning to Rank}

To cast UM as an L2R problem, we first determine appropriate relevance values for each of the UM performance measures presented in Table 2 . These relevance values allow us to use existing L2R performance measures in combination with an L2R technique such as LambdaMART to learn a ranking. Next, as an alternative to using existing L2R measures, we propose a new metric for UM with $\mathrm{L} 2 \mathrm{R}$, called the promoted cumulative gain (PCG), which can be used to learn a ranking that directly optimizes the AUUC.

\subsubsection{Relevance Values for UM with $L 2 R$}

In $\mathrm{UM}$, an instance belongs to either the treatment group $(t=1)$ or the control group $(t=0)$. As shown in Table 2, for evaluating uplift models, we can rely on separate and joint performance measures, depending on whether a ranking is computed for each group separately or for one joint group. Likewise in L2R, one or multiple queries can be considered.
To be more specific, for L2R in the separate UM setting, we consider both the treatment and control groups as separate queries, i.e., an L2R technique will be run to optimize the rankings of the two queries separately. In the joint UM setting, only one query is considered, covering instances of both the treatment and control groups.

We now determine relevance values for each of the UM performance measures from Table 2 . The relevance value of an instance takes over the role of $\hat{\tau}(x)$ which is used in traditional UM. Recall that an instance can belong to one of the following four categories: Treatment Responder (TR), Treatment Non-Responder (TNR), Control Responder (CR) and Control Non-Responder (CNR). To obtain relevance values for each UM performance measure, we check the effect of each of the above categories on their value functions.

Table 3 shows the different value functions and the corresponding relevance values for the separate queries. The effect of a TR or TNR is assessed by looking at the left components of the subtractions in the value functions. A TR increases the overall values obtained, whereas a TNR does not impact the value functions at all. The size of the effect of a TR, however, depends on the value function considered. Similarly, the right components are used to assess the effects of a CR and CNR. An increase in the right components lowers the overall values obtained. Therefore, a negative relevance is assigned to a $C R$, however, the relevance value changes depending on the value function considered. A $\mathrm{CNR}$, on the other hand, does not affect any of the value functions considered.

Table 4 shows the different value functions and the corresponding relevance values for the joint queries. For the relative value function, the relevance values are identical to those for the relative value function for the separate queries. However, for the absolute value functions, TNRs and CNRs no longer have neutral effects on the overall values as the responder counts are rebalanced using the number of treated and control instances among the top- $k$ ranked instances. Consider the Joint Absolute Qini Curve for which the responder count of the control group is rebalanced by multiplication of $\left(N^{T}(\mathcal{D}, k) /\left(N^{C}(\mathcal{D}, k)\right)\right.$. A TNR thus increases this ratio, leading to an increase in the right component of the value function, and hence a lower overall value. A CNR, on the other hand, results in a decreased value for the right component of the value function and hence an increase in the overall value. Quantifying the exact increases and decreases in the overall values, however, is not trivial. Instead, we observe that a TR increases the overall value the most, while a CNR only causes a small increase, a TNR leads to a small decrease and a CR causes a larger decrease in the overall value. We simply encode this relation by using relevance values $3,2,1$ and 0 for a TR, CNR, TNR and CR, respectively. For the Joint Absolute Uplift Curve, a similar analysis gives rise to the same insights (and therefore we do not include it in Table 4).

The various sets of relevance values presented can all be used in combination with any L2R metric that accepts graded relevance values.

\subsubsection{Transforming AUUC into an L2R Metric}

The goal of this section is to come up with an $\mathrm{L} 2 \mathrm{R}$ measure that is most similar to the AUUC, and that can be readily 
TABLE 3: Relevance values for separate queries for each value function definition.

\begin{tabular}{|l|c|c|c|c|}
\hline Value Function & $\begin{array}{c}\text { Treatment Responder } \\
\text { rel }_{T R}\end{array}$ & $\begin{array}{c}\text { Treatment Non-Responder } \\
\text { rel }_{T N R}\end{array}$ & $\begin{array}{c}\text { Control Responder } \\
\text { rel }\end{array}$ & $\begin{array}{c}\text { Control Non-Responder } \\
\text { rel }\end{array}$ \\
\hline$V(p)=R(\mathcal{T}, p|\mathcal{T}|)-R(\mathcal{C}, p|\mathcal{C}|)$ & 1 & 0 & -1 & 0 \\
$V(p)=R(\mathcal{T}, p|\mathcal{T}|)-R(\mathcal{C}, p|\mathcal{C}|)|\mathcal{T}|$ & 1 & 0 & $-\frac{|\mathcal{T}|}{|\mathcal{C}|}$ & 0 \\
$V(p)=\frac{R(\mathcal{T}, p|\mathcal{T}|)}{|\mathcal{T}|}-\frac{R(\mathcal{C}|p| \mathcal{C} \mid)}{|\mathcal{C}|}$ & 1 & 0 & $-\frac{1}{|\mathcal{C}|}$ & 0 \\
\hline
\end{tabular}

TABLE 4: Relevance values for joint queries for each value function definition.

\begin{tabular}{|l|c|c|c|c|}
\hline Value Function & $\begin{array}{c}\text { Treatment Responder } \\
r e l_{T R}\end{array}$ & $\begin{array}{c}\text { Treatment Non-Responder } \\
r e l_{T N R}\end{array}$ & $\begin{array}{c}\text { Control Responder } \\
\text { rel }\end{array}$ & $\begin{array}{c}\text { Control Non-Responder } \\
\text { rel }\end{array}$ \\
\hline$V(k)=\frac{R^{\mathcal{T}}(\mathcal{D}, k)}{|\mathcal{T}|}-\frac{R^{\mathcal{C}}(\mathcal{D}, k)}{\mathcal{C l} \mid}$ & $\frac{1}{\mid \mathcal{T}}$ & 0 & $-\frac{1}{|\mathcal{C}|}$ & 0 \\
$V(k)=R^{\mathcal{T}}(\mathcal{D}, k)-R^{\mathcal{C}}(\mathcal{D}, k) \frac{N^{\mathcal{T}}(\mathcal{D}, k)}{N^{\mathcal{C}}(\mathcal{D}, k)}$ & 3 & 1 & 0 & 2 \\
\hline
\end{tabular}

optimized using existing L2R techniques. To this purpose, we use the relative definitions of the Uplift Curve as the experiment in Section 4.2 shows that these are most robust to differences in group sizes.

Equation 9 shows that the AUUC is a summation of the value function over all possible $k$ values. If we insert the value function definition for the Joint Relative Uplift Curve (Equation 6), we obtain:

$$
A U U C=\sum_{k=1}^{n} V(k)=\sum_{k=1}^{n}\left(\frac{R^{\mathcal{T}}(\mathcal{D}, k)}{|\mathcal{T}|}-\frac{R^{\mathcal{C}}(\mathcal{D}, k)}{|\mathcal{C}|}\right) .
$$

Let us introduce the following helper function $g(i)$ :

$$
g(i)= \begin{cases}0 & \text { if } y=0 \\ 1 /|\mathcal{T}| & \text { if } y=1 \text { and } t=1 \\ -1 /|\mathcal{C}| & \text { if } y=1 \text { and } t=0\end{cases}
$$

where the three cases are mutually exclusive and cover all possible assignment combinations for $y$ and $t$.

Recall from Section 2.2 that $R^{\mathcal{T}}(\mathcal{D}, k)$ and $R^{\mathcal{C}}(\mathcal{D}, k)$ denote the number of treated $(t=1)$ and control $(t=0)$ responders $(y=1)$ among the top- $k$ ranked instances, respectively. As these quantities are obtained by a summation over the top- $k$ instances, Equation 18 can be reformulated in terms of $g(i)$, where $i$ represents a single instance, as follows:

$$
A U U C=\sum_{k=1}^{n} \sum_{i=1}^{k} g(i)=\sum_{i=1}^{n} \sum_{k=i}^{n} g(i)=\sum_{i=1}^{n}(n-i+1) g(i) .
$$

Based on the similarities between the above expression for AUUC and the DCG measure from Equation 15, we introduce a new metric for UM with L2R called the promoted cumulative gain (PCG):

$$
P C G(k)=\sum_{i=1}^{k}(n-i+1) g(i) .
$$

In the DCG measure, each element has a relevance $r e l_{i}$ which is represented by $g(i)$ in our case, and instead of discounting by $1 / \log _{2}(i+1)$ we promote each element by $(n-i+1)$. Finally, along the lines of the DCG measure, PCG allows optimizing the ranking of the top- $k$ instead of the full dataset as well.
A similar analysis can be made for the separate setting, i.e., when the treatment and control groups are ranked separately, based on the Separate Relative Uplift Curve. The PCG can then be obtained as follows (see Appendix A for details):

$$
\begin{aligned}
P C G\left(k_{\mathcal{T}}, k_{\mathcal{C}}\right) & =P C G_{\mathcal{T}}\left(k_{\mathcal{T}}\right)+P C G_{\mathcal{C}}\left(k_{\mathcal{C}}\right) \\
& =\sum_{i=1}^{k_{\mathcal{T}}}(|\mathcal{T}|-i+1) g(i)+\sum_{i=1}^{k_{\mathcal{C}}}(|\mathcal{C}|-i+1) g(i)
\end{aligned}
$$

with $k_{\mathcal{T}}=p|\mathcal{T}|$ and $k_{\mathcal{C}}=p|\mathcal{C}|$ for some percentage $p$. Hence, the PCG can be computed on both subsets separately and summed up. In an L2R setup, that means (i) creating two queries, one for the treatment and one for the control group, (ii) using the PCG measure for each query, and (iii) aggregating the resulting PCG values. Note that the $k$ is different for each query, which requires a small modification to the learning systems.

\subsubsection{Summary}

In this section we summarize the different steps needed to use L2R for UM.

Step 1. Choose one of the two settings: the separate or joint setting. Note that the latter is closer to how uplift models are to be used in practice as producing a ranking for new data implies that none of these new instances has yet been treated.

Step 2. Select a target UM evaluation measure from Table 2 (conditional on the choice made in Step 1) or use PCG (see Section 3.2.2) to assign relevance values to (training) instances (see Tables 3 and 4 and Section 3.2.2).

Step 3. Use an existing L2R technique such as LambdaMART to optimize the full ranking or the top- $k$ according to a selected L2R metric, for either one or two queries, depending on the choice made in Step 1.

\section{EXPERIMENTS}

In this section, we present several experiments to investigate whether and how L2R techniques can be successfully used in the context of UM. In Section 4.1, we first provide information on the datasets and software used. Section 4.2 
covers the first experiment in which we analyze the differences between the value function definitions of the UM performance measures (Table 2) through simulation. This experiment provides useful insights to guide the selection of a target UM evaluation measure for UM with L2R (see Step 2 in Section 3.2.3). In Section 4.3, we compare the performance of a traditional pointwise UM approach, i.e., the flipped label approach, to those of similar listwise L2R approaches. Section 4.4 looks at how the use of different relevance values than those presented in Tables 3 and 4 for a specified target UM evaluation measure affects the performance of the L2R approach (see Step 2 in Section 3.2.3). In Section 4.5 , we investigate the effectiveness of optimizing rankings for the top- $k$ instead of the full dataset (see Step 3 in Section 3.2.3). Finally, in Section 4.6 we compare the performance of the best performing L2R setup to state-ofthe-art UM techniques.

\subsection{Experimental Setup}

\subsubsection{Datasets}

We use three publicly available datasets originating from randomized controlled trials (RCT) for the experiments. An overview of the characteristics of the datasets is provided in Table 5. The first dataset is part of the Information $R$ package ${ }^{1}$. The data relates to a marketing campaign in the insurance industry and the response variable indicates whether or not a purchase happened. The second dataset is published on the website MineThatData ${ }^{2}$ and contains data on an e-mail marketing campaign concerning clothing merchandise. The dataset includes three response variables: 'visit', 'purchase' and 'conversion'. The first two are binary variables, while the third is a numerical response variable that represents the amount of money spent. The dataset includes 64,000 observations with $1 / 3$ targeted with an email campaign concerning men's clothing, $1 / 3$ targeted with an e-mail campaign concerning women's clothing and $1 / 3$ not targeted. For this dataset, in line with [17] to facilitate comparison, the 'visit' variable is selected as the response variable of interest and the selected treatment is the e-mail campaign for women's clothing (reducing the dataset to 42,693 observations). The last dataset is obtained from the Criteo $\mathrm{AI} \mathrm{Lab} 3$ [12] and contains data resulting from several incrementality tests in advertising. The dataset consists of more than 25 million observations, but due to computational reasons, a random subsample of $0.1 \%$ is used, reducing the dataset size to 25,310 observations.

\subsubsection{Software}

The L2R technique employed in the experiments is LambdaMART, for which we use the implementation from the open-source RankLib package ${ }^{4}$ which is implemented in Java. As LambdaMART relies on gradient boosted trees (see Section 3), for fair comparison, we also use gradient boosted trees for all traditional UM approaches. For this, we rely on the implementation in the xgboost $\mathrm{R}$ package [47].

1. https://cran.r-project.org/web/packages/Information/index.html 2. https://blog.minethatdata.com/2008/03/minethatdata-e-mailanalytics-and-data.html

3. https://ailab.criteo.com/criteo-uplift-prediction-dataset/

4. https://sourceforge.net/p/lemur/wiki/RankLib/
Random stratified sampling was applied to the treatment and control groups of each dataset to split them into $80 \%$ training and $20 \%$ test data while preserving the overall response rate within each group. All reported results are on the test set, unless explicitly stated otherwise. Parameter tuning was done upfront based on the performance on a validation set containing $20 \%$ of the data and hyperparameters are kept identical for all experiments in the paper (500 trees and a learning rate of 0.01). Each experiment is repeated 10 times. Reported results are averages of these different runs, and in plots we visualize the range between the minimum and the maximum value as a shaded area.

\subsection{Experiment 1: Comparing UM Performance Mea- sures through Simulation}

To optimize L2R techniques for UM, we need to select a UM evaluation measure from Table 2. From the table, one can see that the normalization used to account for possible differences in treatment and control group sizes is a differentiating factor. To better understand these differences, we simulate data for three different scenarios with regard to the sizes of the treatment and control groups, produce a ranking for each scenario, and compare how these rankings are evaluated by the different UM evaluation measures presented in Table 2.

The simulation consists of two populations: the treatment group with a response rate of $7 \%$ and the control group with a response rate of $5 \%$. Each instance is assigned a value for $\hat{\tau}(x)$ between 0 and 1 depending on the category the instance is in (i.e., TR, TNR, CR or CNR). To simulate uplift, we use the following procedure: for a TR and CNR, we uniformly sample from the interval $[0.2,1.0]$, while for a CR and TNR we sample from the interval $[0.0,0.8]$. These intervals ensure that TR and CNR instances will appear higher in the overall ranking than CR and TNR instances. In a next step, we sample from both treatment and control groups to create three scenario's: (1) a balanced setting with an equal number of instances in both groups $(|\mathcal{T}|=|\mathcal{C}|)$, (2) an imbalanced setting with a larger treatment group $(|\mathcal{T}|=9|\mathcal{C}|)$ and (3) an imbalanced setting with a larger control group $(|\mathcal{C}|=9|\mathcal{T}|)$.

Because each instance is assigned a value for $\hat{\tau}(x)$, we can create a ranking for each scenario and compare how these rankings are evaluated by the different UM metrics. To this end, we visualize the different curves (and corresponding baselines) obtained for both the absolute and relative definitions in Figures 2 and 3, respectively. We split up the analysis for the absolute and relative definitions as they have different units of measurement.

For the absolute definitions, we observe in Figure 2 that the Qini curves behave quite similar for the separate and joint settings at first sight. However, a closer inspection shows that higher a Qini Curve, and thus a higher AUUC, is obtained for the separate setting. For the Qini curves, AUUC values are all positive but differ significantly depending on the sizes of the groups. For the Uplift curves, on the other hand, the behavior of the curves in separate and joint setting, as well as for the different scenarios, is very different. Moreover, for the Separate Absolute Uplift Curve, AUUC values turn negative when the control group is significantly 
TABLE 5: Characteristics of datasets used in the experiments.

\begin{tabular}{|l|lll|}
\cline { 2 - 4 } \multicolumn{1}{c|}{} & Information & Hillstrom & Criteo \\
\hline Description & Insurance & Online clothing & Marketing \\
Channel & E-mail & E-mail & Advertisement \\
Total size & 10,000 & 64,000 & $25,309,483$ \\
\# Treatment observations & 4,972 & 21,387 & 21,409 \\
\# Control observations & 5,028 & 21,306 & 3,901 \\
\# Variables & 68 & 10 & 14 \\
Response variable (binary) & Purchase & Visit & Visit \\
Treatment-to-control size ratio & $0.99: 1$ & $1: 1$ & $5.48: 1$ \\
Treatment positive rate & $20.37 \%$ & $15.14 \%$ & $4.41 \%$ \\
Control positive rate & $19.55 \%$ & $10.62 \%$ & $2.61 \%$ \\
Uplift initial campaign & $0.82 \%$ & $4.52 \%$ & $1.80 \%$ \\
\hline
\end{tabular}

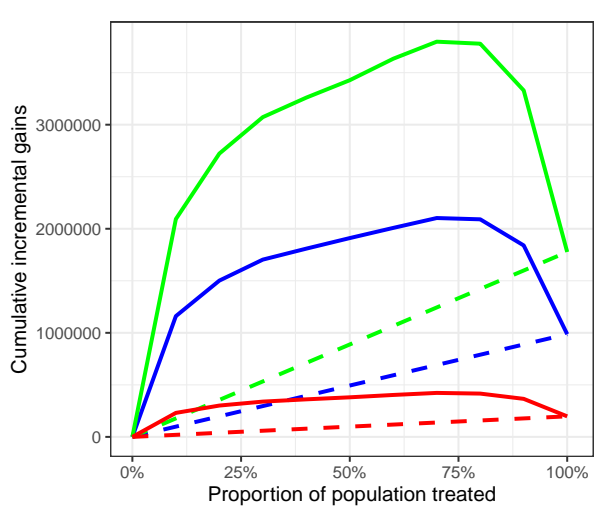

(a) Separate Absolute Qini Curve

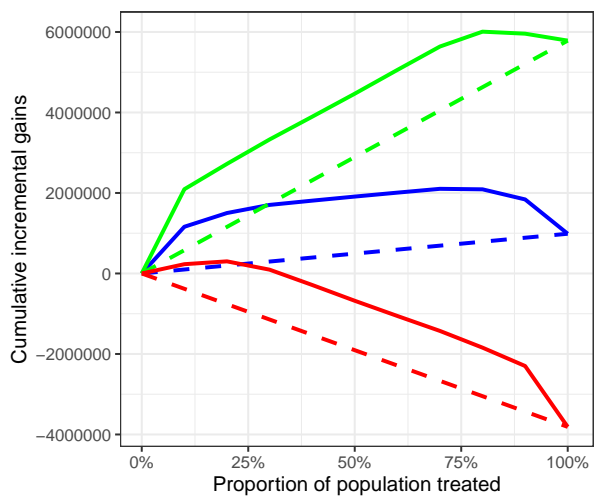

(c) Separate Absolute Uplift Curve

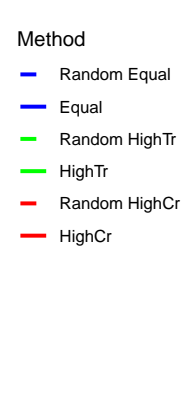

- Random Equal

- Equal

- Random HighTr

- HighTr

- Random HighCr - HighCr

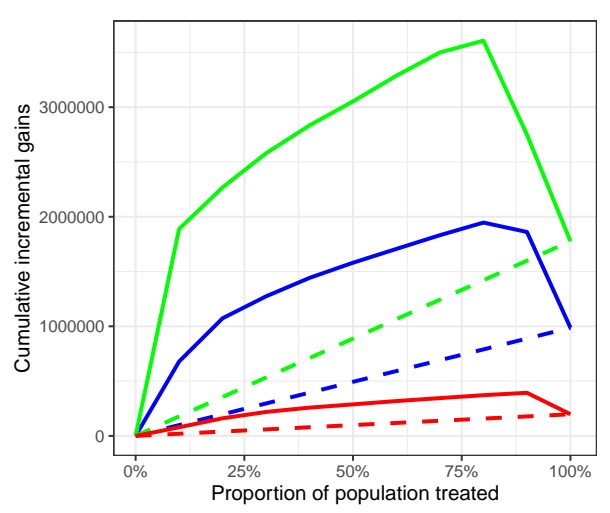

Method

- Random Equal

- Equal

- Random Hightr

- HighTr

- Random HighCr

- HighCr

Fig. 2: Experiment 1. Simulation of the absolute curves in both separate and joint setting. In each figure, the baseline of each respective scenario is represented by the dashed line. In Figure $2 \mathrm{~d}$ the baselines for the different scenarios are equal.

larger than the treatment group, which is due to the fact that there is no normalization between the treatment and control groups for this UM performance measure.

For the two relative definitions in Table 2, we observe in Figure 3 that there are no differences in the curves (and the corresponding AUUC values) for the different scenarios in the separate setting. Recall from Section 2.2 that the Separate Relative Uplift Curve is equivalent to the Separate Absolute Qini Curve up to the constant scaling factor $|\mathcal{T}|$, which implies that the curves in Figure 3a are rescaled versions of the ones in Figure 2a, and that the same ranking would be obtained when these measures are used for optimization. For the joint setting in Figure 3b, in contrast to the separate setting, we observe that a different scenario results in a shift of the Uplift Curve. A possible explanation is that one group is overrepresented in the top fraction of the ranking, causing the uplift to be one-sided. However, the AUUC values are very similar. Finally, by comparing the separate and joint settings for the balanced scenario in Figure 3c, we observe that we get a higher Uplift Curve for the separate setting, and thus a higher AUUC. This is also true for the other scenarios. The reason is that, in the separate setting, the number of TR instances in the top fraction is much higher than in the joint setting, in which we see relatively more CNR instances in the top fraction, which heavily influences the Uplift curves in the early parts of the plot.

Given the above comparisons for the simulated rankings, we consider the relative definitions to be more robust to 


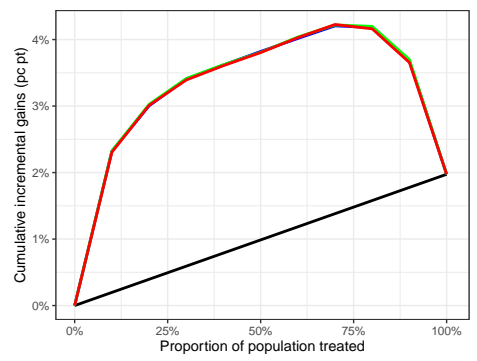

(a) Separate Relative Uplift Curve

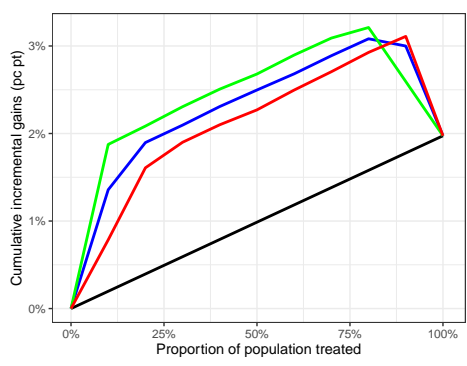

(b) Joint Relative Uplift Curve

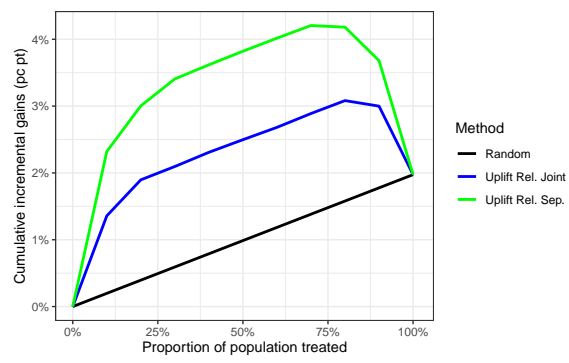

(c) Sep. Rel. vs. Joint Rel. Uplift Curve

Fig. 3: Experiment 1. Simulation of the relative curves in both separate and joint setting.

differences in treatment and control group sizes as these $\mathrm{UM}$ evaluation measures are (rather) stable under the three different scenarios. As this can be considered a useful property, in the experiments that follow, we use the relative UM measures, both in separate and joint setting.

\subsection{Experiment 2: Pointwise UM vs. Listwise L2R}

Recall that the flipped label approach can be considered as a pointwise L2R method in which a single model has to rank TRs higher than TNRs and CNRs higher than CRs. Hence, both TRs and CNRs are assigned a new label $z=1$, whereas the rest is labeled $z=0$ with $z \in\{0,1\}$. For the flipped label approach, there is thus no need to separate instances in different groups. In this experiment, we compare this baseline uplift model with the listwise L2R technique LambdaMART. In this regard, recall from Section 4.1.2 that we use gradient boosted trees as learning algorithm for all traditional UM approaches to ensure a fair comparison with LambdaMART.

In the flipped label approach, no distinction is made between TRs and CNRs, and TNRs and CRs. In L2R context, this is similar to the separate setting as the relevant instances of each query have no relation to each other. Therefore, we compare the flipped label approach to $L 2 R$ by using LambdaMART for two separate queries, one for the treatment group and one for the control group. As for the relevance values we use binary values in accordance with the labels used in the flipped label approach.

Typically, LambdaMART is used to optimize only the top- $k$ of the ranking (as users of for example search engines typically only focus on the first $k$ results), however, in order to compare with the baseline uplift model we use LambdaMART to optimize over all instances. Therefore, in this experiment, $k$ is set equal to the number of training instances in the group considered (either treatment or control). Finally, we use LambdaMART to optimize four different metrics: MAP, DCG, NDCG and PCG.

Figure 4 shows the relative Uplift curves for the baseline uplift model and the different LambdaMART setups for the three different datasets. On the Information dataset (Figure 4a), the pointwise UM approach performs better than the standard LambdaMART setups based on DCG, NDCG and MAP. However, for LambdaMART optimized with our PCG metric, the Uplift curve shows higher cumulative incremental gains in the early parts of the plot when compared to the pointwise UM approach. On the Hillstrom dataset
(Figure 4b), LambdaMART with MAP, DCG, NDCG and PCG perform equally well compared to the pointwise UM approach, and perform significantly better for proportions of the population treated above $40 \%$. Finally, on the Criteo dataset (Figure 4c), all techniques achieve high cumulative incremental gains in the early parts of the plot (first 10\% of the population treated). However, for proportions of the population treated between $10 \%$ and $40 \%$, the pointwise UM approach performs worse than the listwise L2R approaches.

We further analyze the results by examining the AUUC values presented in Table 6 . The pointwise UM approach performs better than the listwise L2R approaches on the Information dataset with only LambdaMART PCG coming close. However, the listwise L2R approaches marginally perform better on both the Hillstrom and Criteo datasets.

TABLE 6: Experiment 2. AUUC values of the Separate Relative Uplift Curve for the baseline uplift model and the different LambdaMART setups. Values in bold: best value on that dataset.

\begin{tabular}{|l|l|l|l|}
\cline { 2 - 4 } \multicolumn{1}{c|}{} & \multicolumn{3}{c|}{ Separate Relative AUUC } \\
\hline Technique & Information & Hillstrom & Criteo \\
\hline Flipped label approach & $\mathbf{0 . 0 2 0 5 2}$ & 0.02858 & 0.01479 \\
\hline LambdaMART MAP & 0.01237 & 0.03038 & 0.01556 \\
LambdaMART DCG & 0.01520 & 0.02960 & 0.01522 \\
LambdaMART NDCG & 0.00935 & 0.03032 & 0.01523 \\
LambdaMART PCG & 0.01938 & $\mathbf{0 . 0 3 0 7 7}$ & $\mathbf{0 . 0 1 5 7 8}$ \\
\hline
\end{tabular}

In summary, the results of this experiment indicate that listwise $L 2 R$ approaches can be viable alternatives to pointwise UM approaches. Further, we observe that, for the datasets considered, using the proposed PCG metric to optimize LambdaMART always leads to a better result compared to using one of the other L2R metrics considered.

\subsection{Experiment 3: Different Sets of Relevance Values}

In this experiment, we investigate how the use of different relevance values than those presented in Tables 3 and 4 for a specified target UM evaluation measure affects the performance of the L2R approach. In this regard, recall that in Section 3.2.1 we determined appropriate relevance values for each UM evaluation measure. Based on these values, presented in Tables 3 and 4, and the relevance values used for the flipped label approach in Section 4.3, we can create the four different sets of relevance values presented in Table 7 . We test these different relevance value sets for both the 
1

2

3

4

5

6

7

8

9

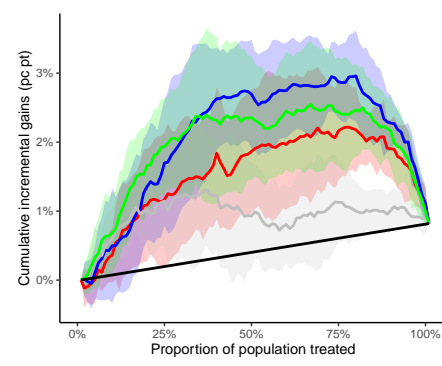

(a) Information
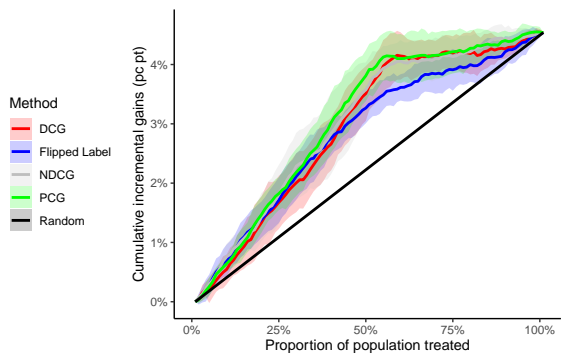

(b) Hillstrom
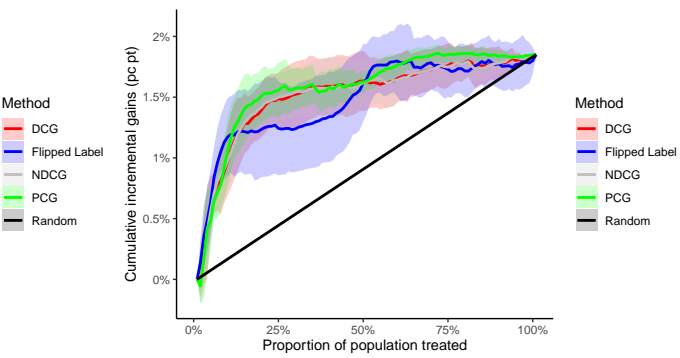

(c) Criteo

Fig. 4: Experiment 2. Relative Uplift curves in separate setting. Black color is used to represent random treatment assignment, blue represents the baseline uplift model and the other colors represent the different LambdaMART setups.

separate and joint setting and use DCG, NDCG and PCG as optimization metrics for LambdaMART. These metrics are chosen because they can handle graded relevance values.

TABLE 7: Experiment 3. Different sets of relevance values.

\begin{tabular}{|l|c|c|c|c|}
\hline Set & TR & TNR & CR & CNR \\
\hline Absolute relevance 1 & 1 & 0 & 0 & 1 \\
Absolute relevance 2 & 1 & 0 & -1 & 0 \\
Absolute relevance 3 & 3 & 1 & 0 & 2 \\
Relative relevance & $\frac{1}{|\mathcal{T}|}$ & 0 & $-\frac{1}{|\mathcal{C}|}$ & 0 \\
\hline
\end{tabular}

For the use of separate queries, the results are reported in Table 8 in terms of AUUC values of the Separate Relative Uplift Curve. We observe that in all possible settings, PCG consistently performs best when compared to other metrics. On the Information dataset, the NDCG performs significantly worse compared to the other approaches, with the 'absolute relevance 3 ' setting being the exception, however, on the Hillstrom and Criteo datasets, the performance of the NDCG metric is fairly equal to that of the DCG metric. More interestingly, despite the fact that PCG with 'relative relevance' labels is exactly the same as optimizing AUUC, using one of the absolute relevance label sets performs marginally better on the Information and Hillstrom datasets.

For the use of a joint query, the results are reported in Table 9 in terms of AUUC values of the Joint Relative Uplift Curve. Also in this setting, we observe that PCG consistently outperforms the other metrics. Furthermore, now an absolute relevance value set performs best for all datasets and metrics (while also here the use of 'relative relevance' labels in combination with PCG is equivalent to optimizing AUUC). Finally, also note that the NDCG shows improved and nearly equal results to those of DCG on all datasets, including the Information dataset. This is as expected, as in theory, NDCG and DCG should produce equal results when there is only one query.

In summary, the above results are somewhat surprising. While they do confirm that PCG is better suited to the task, the use of less theoretically motivated relevance values is shown to be able to produce good results too.

\subsection{Experiment 4: Optimizing Rankings for the Top- $k$}

In UM, as in information retrieval, often only the top$k$ fraction of the ranking is of interest to the user. For example, in marketing or churn prediction, the size of the fraction is determined by the campaign budget. One of the properties of LambdaMART and other L2R systems is their ability to optimize for the top- $k$ specifically. Current UM techniques, however, all aim to optimize the entire ranking. Therefore, in this experiment, we investigate whether specifically optimizing for a specific top- $k$ by using L2R can be successfully applied to UM. In the experiments above, we always optimized the rankings for the entire datasets by setting $k$ equal to the number of training instances in the group considered (either treatment, control or both).

In this experiment, we optimize LambdaMART for $k$ values equal to $10 \%, 30 \%$ and $50 \%$. For each of these $k$ values, we then check the Uplift curves and AUUC values for both separate and joint settings. Additionally, we compare the results with our previous experiments in which we optimized the rankings for the entire datasets. We present the results of LambdaMART optimized with our PCG metric. We further use for relevance values the 'relative relevance' set as this is closest to directly optimizing AUUC. We also carried out this experiment with the DCG and NDCG metrics used for optimization, and with relevance values as in the 'absolute relevance 3 ' setting, however, these settings provided similar insights and therefore the results are not reported for brevity.

For each model, we visualize the Uplift curves from both an optimization and generalization perspective. The optimization perspective shows the results of the models when evaluated on the training set (which is only indicative of the effect of training). The generalization perspective shows the results of the models when evaluated on the test set (the proper way to evaluate the model). The results are shown in Figures 5 and 6 for the separate and joint settings, respectively.

The plots from an optimization perspective for both the separate and joint setting show that the L2R techniques can optimize for a specific $k$ value. We see that these Uplift curves have change points, after which their behavior changes. These effects are most clearly visible in the joint setting, where it is especially pronounced for $k=10 \%$, for which we observe peaks around the $10 \%$ marks, followed by declines in the cumulative incremental gains.

When investigating the effect of optimizing for a specific top- $k$ on the test set, i.e., how well the results generalize to new data, the figures provide a less clear answer. Table 10 
TABLE 8: Experiment 3. AUUC values of the Separate Relative Uplift Curve. Values in bold: best value in that column. Underlined values: best value on that dataset.

\begin{tabular}{|l|l|l|l||l|l|l||l|l|l|}
\cline { 2 - 9 } \multicolumn{1}{c|}{} & \multicolumn{3}{c||}{ Information } & \multicolumn{3}{c||}{ Hillstrom } & \multicolumn{3}{c|}{ Criteo } \\
\hline Set & DCG & NDCG & PCG & DCG & NDCG & PCG & DCG & NDCG & PCG \\
\hline Absolute relevance 1 & $\mathbf{0 . 0 1 5 2 0}$ & 0.00935 & $\mathbf{0 . 0 1 9 3 8}$ & 0.02960 & $\mathbf{0 . 0 3 0 3 2}$ & $\mathbf{0 . 0 3 0 7 7}$ & 0.01522 & 0.01523 & 0.01578 \\
Absolute relevance 2 & $\mathbf{0 . 0 1 5 2 0}$ & 0.00678 & $\mathbf{0 . 0 1 9 3 8}$ & 0.02960 & 0.02893 & $\mathbf{0 . 0 3 0 7 7}$ & 0.01522 & $\mathbf{0 . 0 1 5 5 5}$ & 0.01578 \\
Absolute relevance 3 & $\mathbf{0 . 0 1 5 2 0}$ & $\mathbf{0 . 0 1 5 2 4}$ & $\mathbf{0 . 0 1 9 3 8}$ & 0.02960 & 0.02953 & $\mathbf{0 . 0 3 0 7 7}$ & 0.01522 & 0.01538 & 0.01578 \\
Relative relevance & 0.01382 & 0.00677 & $\mathbf{0 . 0 1 8 2 9}$ & $\mathbf{0 . 0 2 9 6 1}$ & 0.02893 & $\mathbf{0 . 0 3 0 5 5}$ & $\mathbf{0 . 0 1 5 4 9}$ & $\mathbf{0 . 0 1 5 5 5}$ & $\mathbf{0 . 0 1 6 0 1}$ \\
\hline
\end{tabular}

TABLE 9: Experiment 3. AUUC values of the Joint Relative Uplift Curve. Values in bold: best value in that column. Underlined values: best value on that dataset.

\begin{tabular}{|l|l|l|l||l|l|l||l|l|l|}
\cline { 2 - 9 } \multicolumn{1}{c|}{} & \multicolumn{3}{c||}{ Information } & \multicolumn{3}{c||}{ Hillstrom } & \multicolumn{3}{c|}{ Criteo } \\
\hline Set & DCG & NDCG & PCG & DCG & NDCG & PCG & DCG & NDCG & PCG \\
\hline Absolute relevance 1 & 0.01396 & 0.01452 & 0.01940 & 0.02957 & 0.02957 & 0.03002 & 0.01497 & 0.01494 & 0.01469 \\
Absolute relevance 2 & 0.01101 & 0.01116 & 0.01536 & 0.02935 & 0.02935 & 0.03051 & $\mathbf{0 . 0 1 6 0 7}$ & $\mathbf{0 . 0 1 6 0 7}$ & $\mathbf{0 . 0 1 6 6 9}$ \\
Absolute relevance 3 & $\mathbf{0 . 0 1 5 6 3}$ & $\mathbf{0 . 0 1 4 7 3}$ & $\mathbf{0 . 0 2 3 0 0}$ & $\mathbf{0 . 0 2 9 6 8}$ & $\mathbf{0 . 0 2 9 6 8}$ & $\mathbf{0 . 0 3 0 6 3}$ & 0.01568 & 0.01568 & 0.01536 \\
Relative relevance & 0.01052 & 0.01031 & $\mathbf{0 . 0 1 5 7 3}$ & 0.02954 & 0.02954 & $\mathbf{0 . 0 3 0 2 7}$ & 0.01541 & 0.01543 & 0.01554 \\
\hline
\end{tabular}

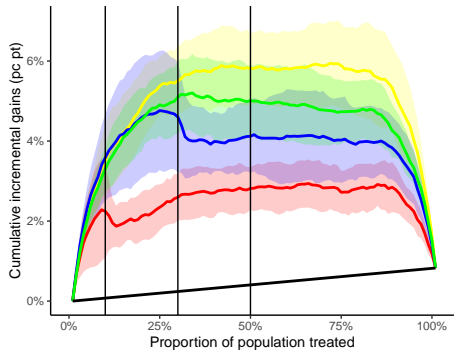

(a) Information - optimization

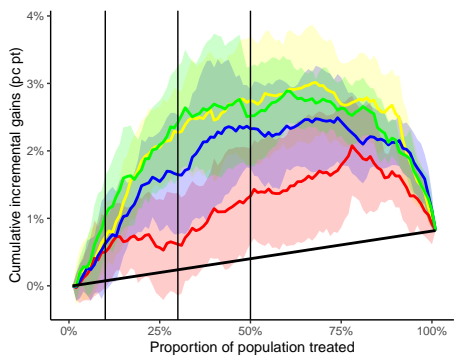

(d) Information - generalization

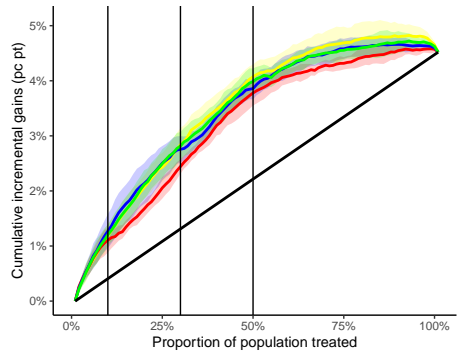

(b) Hillstrom - optimization
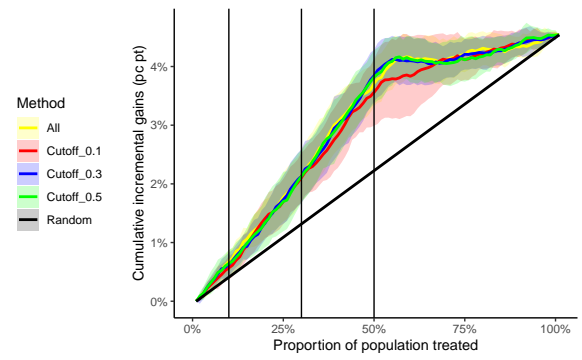

(e) Hillstrom - generalization

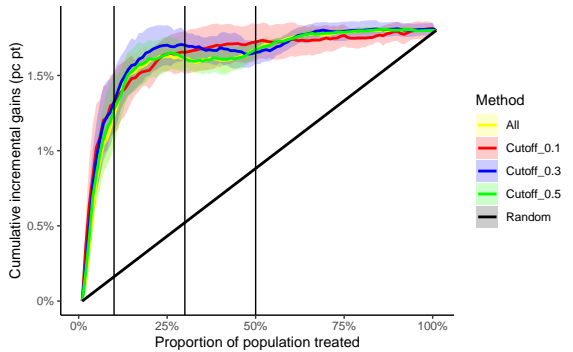

(c) Criteo - optimization
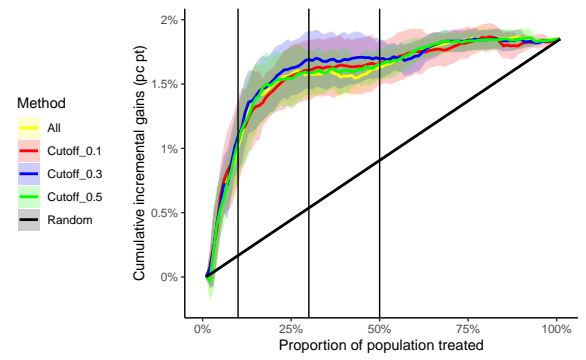

(f) Criteo - generalization

Fig. 5: Experiment 4. Relative Uplift curves in separate setting at multiple cutoffs. The first row is tested on the training set. The second row is tested on the test set.

provides a closer look by presenting the AUUC values of the relative Uplift curves from the generalization perspective at the different $k$ values. We observe that optimizing for a specific $k$ value shows better performance than optimizing for the entire dataset only in some cases (mainly on the Criteo dataset). However, in general, we see that there is no direct relation between optimizing for a specific $k$ value and the results obtained for that value on the test set, nor for other values. Hence, this is a negative result: while learning to optimize AUUC up to a specific cutoff is possible, there is no significant benefit compared to optimizing for the entire dataset (and total AUUC) on the three datasets used in our experiments.

\subsection{Experiment 5: LambdaMART PCG vs. State-of-the- art UM Techniques}

In this last experiment we compare the L2R LambdaMART technique, used in combination with our PCG metric, to state-of-the-art UM techniques. In experiment 2 (Section 4.3), we focused on comparing the performance of different LambdaMART setups to that of the flipped label approach. By contrast, in this experiment we focus on comparing the performance of the best LambdaMART setup to that of the multiple state-of-the-art UM techniques. Next to the flipped label approach, we also consider the dummy treatment approach, the two model approach, and the uplift random forest (see Section 2.1). Of all these techniques, the uplift random forest shows the most consistent performances according to a previous benchmark study [5]. For 


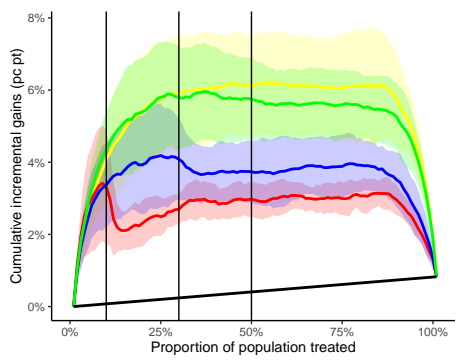

(a) Information - optimization

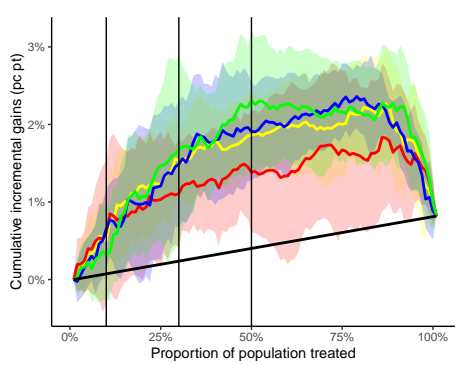

(d) Information - generalization

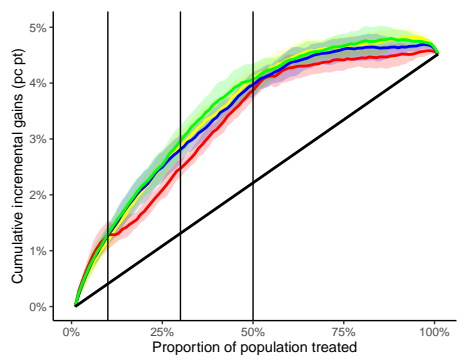

(b) Hillstrom - optimization
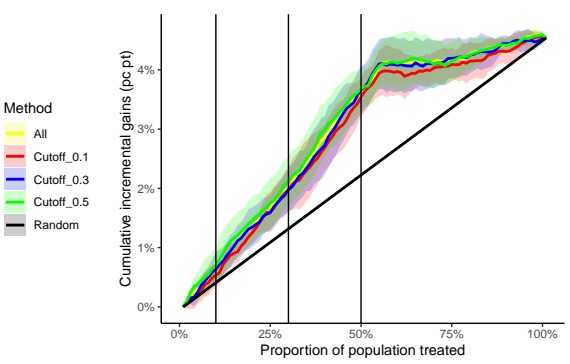

(e) Hillstrom - generalization

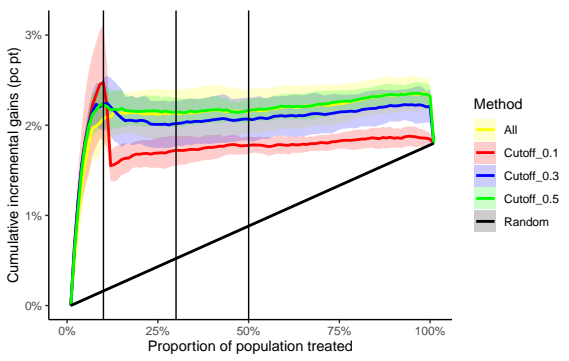

(c) Criteo - optimization

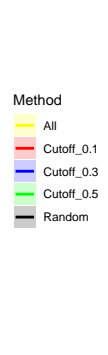

Fig. 6: Experiment 4. Relative Uplift curves in joint setting at multiple cutoffs. The first row is tested on the training set. The second row is tested on the test set.

TABLE 10: Experiment 4. AUUC values of relative Uplift curves at multiple cutoffs on the test set. The left half is in the separate setting and the right half in the joint setting. Values in bold: performance of PCG @ k is higher than PCG @ 100\% in that cell. Underlined values: best value in that cell.

\begin{tabular}{|c|c|c|c|c|c|c|c|c|c|}
\hline & \multicolumn{4}{|c|}{ Separate Relative AUUC at cutoff } & \multicolumn{4}{|c|}{ Joint Relative AUUC at cutoff } \\
\hline & & $10 \%$ & $30 \%$ & $50 \%$ & $100 \%$ & $10 \%$ & $30 \%$ & $50 \%$ & $100 \%$ \\
\hline \multirow{4}{*}{ Information } & PCG @ 100\% & 0.00037 & 0.00384 & 0.00900 & $\underline{0.02178}$ & 0.00034 & $\underline{0.00271}$ & 0.00616 & 0.01573 \\
\hline & PCG@ $10 \%$ & 0.00026 & 0.00159 & 0.00368 & $\overline{0.01150}$ & 0.00040 & $\overline{0.00232}$ & 0.00495 & 0.01264 \\
\hline & PCG@30\% & 0.00030 & 0.00299 & 0.00731 & 0.01819 & 0.00030 & 0.00246 & 0.00611 & 0.01608 \\
\hline & PCG@ @ 50\% & $\underline{0.00049}$ & $\underline{0.00419}$ & $\underline{0.00942}$ & 0.02143 & 0.00021 & 0.00257 & $\underline{0.00646}$ & $\underline{0.01690}$ \\
\hline \multirow{4}{*}{ Hillstrom } & PCG @ 100\% & $\overline{0.00037}$ & $\overline{0.00332}$ & $\overline{0.00947}$ & 0.03060 & 0.00037 & 0.00319 & $\overline{0.00905}$ & $\overline{0.03027}$ \\
\hline & PCG@ $@ 10 \%$ & 0.00032 & $\overline{0.00318}$ & $\overline{0.00901}$ & $\overline{0.02980}$ & 0.00029 & 0.00296 & 0.00853 & 0.02920 \\
\hline & PCG @ 30\% & 0.00037 & 0.00323 & 0.00934 & 0.03045 & 0.00035 & 0.00313 & 0.00887 & 0.03001 \\
\hline & PCG @ 50\% & 0.00038 & 0.00321 & 0.00935 & 0.03043 & 0.00043 & $\underline{0.00347}$ & 0.00950 & $\underline{0.03073}$ \\
\hline \multirow{4}{*}{ Criteo } & PCG @ 100\% & 0.00059 & 0.00354 & 0.00671 & 0.01575 & 0.00088 & 0.00380 & 0.00691 & 0.01554 \\
\hline & PCG@10\% & $\underline{0.00063}$ & 0.00353 & 0.00681 & 0.01577 & 0.00087 & 0.00392 & 0.00725 & 0.01599 \\
\hline & PCG @ 30\% & $\overline{0.00062}$ & $\underline{0.00368}$ & $\underline{0.00706}$ & $\underline{0.01608}$ & $\underline{0.00092}$ & $\overline{0.00383}$ & $\overline{0.00689}$ & $\overline{0.01545}$ \\
\hline & PCG @ 50\% & 0.00058 & 0.00353 & 0.00676 & 0.01580 & 0.00088 & 0.00377 & 0.00684 & 0.01533 \\
\hline
\end{tabular}

the L2R setup, we opt for LambdaMART with PCG and 'relative relevance' values in a separate setting. We also carried out the experiment with the 'absolute relevance 3 ' values, however, we do not report the results as they lead to the same conclusions.

Figure 7 shows the relative Uplift curves in separate setting for the three datasets. We observe that most UM techniques perform better than LambdaMART PCG on the Information dataset, with the two model approach being the best performing technique. However, on the Hillstrom and Criteo datasets, LambdaMART PCG shows improved performance over the UM techniques. On the Hillstrom dataset, LambdaMART PCG achieves $4 \%$ cumulative incremental gains as the only technique at $50 \%$ of the population treated, whereas the other techniques only achieve this when treating almost everyone. For the Criteo dataset, we observe that the flipped label approach performs well when treating only $10 \%$ of the population, however, for higher treatment percentages LambdaMART PCG outperforms the UM techniques. Also notice that some UM techniques even perform worse than the baseline for very high treatment percentages.

We further analyze the results by examining the AUUC values presented in Table 11. On the Information dataset, all UM techniques perform better in terms of AUUC than LambdaMART PCG. However, on both the Hillstrom and Criteo datasets, LambdaMART PCG performs better in terms of AUUC than the UM techniques. Additionally, we present the AUUC results for the relative joint setting, however, based on the results the same conclusions can be drawn. In summary, these results thus show that the L2R LambdaMART technique can compete with existing stateof-the-art UM techniques, and in some scenarios can even do better. 


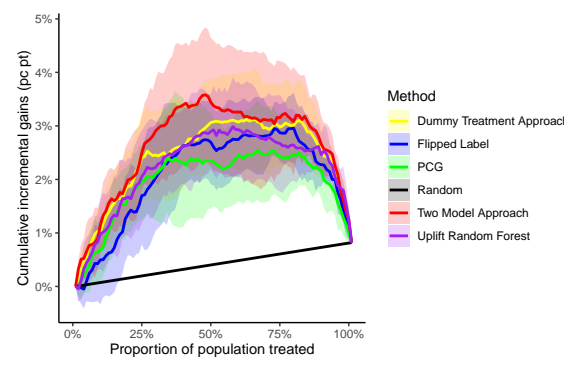

(a) Information

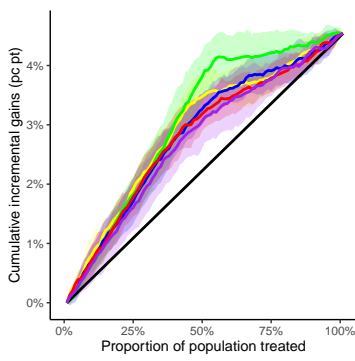

(b) Hillstrom

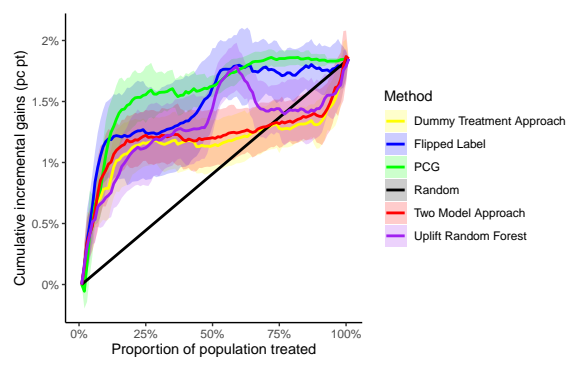

(c) Criteo

Fig. 7: Experiment 5. Relative Uplift curves in separate setting. Comparison of LambdaMART PCG (and 'relative relevance' values) with different UM techniques.

TABLE 11: Experiment 5. AUUC values of the Separate and Joint Relative Uplift Curve for LambdaMART PCG and the different UM techniques. Values in bold: best value on that dataset.

\begin{tabular}{|l|l|l|l||l|l|l|}
\cline { 2 - 7 } \multicolumn{1}{c|}{} & \multicolumn{3}{c||}{ Separate Relative AUUC } & \multicolumn{3}{c|}{ Joint Relative AUUC } \\
\hline Technique & Information & Hillstrom & Criteo & Information & Hillstrom & Criteo \\
\hline LambdaMART PCG & 0.01829 & $\mathbf{0 . 0 3 0 5 5}$ & $\mathbf{0 . 0 1 6 0 1}$ & 0.01791 & $\mathbf{0 . 0 3 0 5 7}$ & $\mathbf{0 . 0 1 6 7 7}$ \\
\hline Dummy treatment approach & 0.02392 & 0.02935 & 0.01165 & 0.02283 & 0.02950 & 0.01181 \\
Two model approach & $\mathbf{0 . 0 2 6 1 0}$ & 0.02820 & 0.01213 & $\mathbf{0 . 0 2 5 7 8}$ & 0.02840 & 0.01224 \\
Flipped label & 0.02052 & 0.02858 & 0.01479 & 0.02050 & 0.02865 & 0.01418 \\
Uplift random forest & 0.02210 & 0.02744 & 0.01287 & 0.02163 & 0.02746 & 0.01174 \\
\hline
\end{tabular}

\section{Discussion}

In our first experiment, we compared different definitions of the Qini and Uplift curves by analyzing performance results on simulated rankings. The results showed that the sizes of the treatment and control groups heavily influence the cumulative incremental gains when expressed in absolute terms. Expressing the cumulative incremental gains in relative terms results in a more robust evaluation. Based on these results, we continued our experiments by focusing on the relative evaluation measures (and their corresponding AUUC values) for both the separate and joint settings, in which we rank the treatment and control groups separately or as one joint group, respectively. Note that in this work we only consider the binary treatment case, i.e., an instance is either treated or not treated. However, this could be extended to multiple treatments by creating a query for each treatment as this is very straightforward in L2R.

In the second experiment, listwise L2R techniques are compared to the pointwise UM flipped label approach in the separate setting. We test LambdaMART with both standard L2R metrics, such as DCG and NDCG, and with our own AUUC-centric metric, PCG. The results show that LambdaMART with PCG performs equal to or better than the flipped label approach which already indicates that listwise L2R techniques can be viable alternatives to pointwise UM techniques.

In the third experiment, we investigated whether the use of different sets of relevance values significantly affects the performance of the L2R approaches. The results of this experiment clearly indicate that our PCG metric outperforms standard L2R metrics in the different settings considered. When optimizing PCG with the 'relative relevance' values, we directly optimize the AUUC. However, the results show that the use of less theoretically motivated relevance values also produces good results, and even marginal improve- ments in performance. In the separate setting, this can be explained by the fact that changing the relevance values does not affect the preference orders. However, in the joint setting, changing the relevance values does affect the preference orders between the four categories (TR, TNR, CR and $\mathrm{CNR}$ ). Future research could investigate the effects of different sets of relevance values. Moreover, this also paves the way to future research on multipartite ranking methods [48] in a UM context.

The fourth experiment investigated the potential of optimizing rankings for the top- $k$ in UM. From an optimization perspective, we often did observe a change in behavior as change points in performance were clearly identified around the $k$ value used in optimization. This effect was observed in both the separate and joint settings, however, it is more distinguishable in the joint setting, indicating that the joint setting is more suitable for optimization. However, these results did not generalize to the test set. This experiment thus indicates that optimizing rankings for top- $k$ fractions of the population is possible, but that there is no significant benefit compared to optimizing for the entire dataset.

Finally, in the fifth experiment, we compared the performance of LambdaMART PCG, which is the best performing L2R setup, to those of existing state-of-the-art UM techniques. The UM techniques show varying performances on all datasets. On the Information dataset, the UM techniques do seem to perform better than LambdaMART PCG. However, on the Hillstrom and Criteo datasets, the results show that LambdaMART PCG outperforms the UM techniques in terms of AUUC. These results indicate that LambdaMART PCG is able to better identify the most impactful instances for smaller proportions of the population treated compared to the UM techniques. Therefore, we can conclude that $\mathrm{L} 2 \mathrm{R}$ techniques can be added to the UM toolbox of techniques. 


\section{Conclusion}

Causal classification models estimate for each instance the causal effect of a treatment on an outcome variable of interest, i.e., the individual treatment effect (ITE). If both treatment and outcome are binary variables, ITE estimates allow one to rank instances from a large positive effect to a large negative effect. In uplift modeling (UM), one is exactly interested in this ranking rather than in the ITE estimates themselves, as the aim is to identify the instances that are most likely to respond as an effect of being treated. Uplift models estimate the ITE and then use it to build a ranking from which a fraction is selected for treatment. On the other hand, Learning to Rank (L2R) techniques comprise techniques specifically designed to optimize the quality of predicted rankings directly, rather than the quality of predicted values that serve to rank instances. This paper explores the possibility of using L2R techniques, more specifically the well-known LambdaMART technique, in a UM context.

Before UM was cast as an L2R problem, an analysis of the current evaluation metrics of UM was done. This analysis shows conflicting definitions in the literature. The main differences among definitions are (1) whether the treatment and control groups are considered as separate groups or as one joint group and (2) whether the cumulative incremental gains are expressed in absolute units or in relative percentages.

Our experiments show that standard $\mathrm{L} 2 \mathrm{R}$ techniques can be viable alternatives to UM techniques by comparing their performances in terms of Area Under the Uplift Curve (AUUC) for two UM metric definitions selected (the Separate Relative Uplift Curve and Joint Relative Uplift Curve). With the promoted cumulative gain (PCG) we have created a new L2R metric which promotes relevance values of instances earlier in the ranking, instead of discounting relevance values of instances further in the ranking. The PCG is exactly the AUUC metric from UM and can be readily used by the LambdaMART L2R technique. Moreover, using the proposed PCG metric to optimize LambdaMART is shown to produce better results in terms of AUUC than using standard L2R metrics and to achieve equal or better results than the baseline uplift model (i.e., the flipped label approach).

This paper also tested the effectiveness of optimizing rankings for the top- $k$ instead of the full dataset in a UM context. Models were trained to optimize their rankings for the top $10 \%, 30 \%, 50 \%$ and $100 \%$. While the results show that learning to optimize rankings for a specific top fraction of the population is possible, from a generalization perspective, no significant benefits can be observed compared to optimizing for the entire dataset.

Finally, with the last experiment we have shown that LambdaMART PCG can compete with existing state-of-theart UM techniques and even lead to improved performances in terms of AUUC. Overall, our results confirm that L2R can be regarded as a viable alternative to the existing UM methodology by focusing on modeling the ranking directly instead of predicting values that are used to produce a ranking. This work brings up new research questions for future research:
- Future research could look into other listwise L2R techniques.

- Further investigating the effects of using different relevance values for CNRs and TNRs is another possibility.

- The potential of multipartite ranking methods [48] could be explored in UM context, which is related to the previous point.

- One of the open questions in UM is the use case of having multiple treatments [21]. The L2R framework readily allows one to plug in multiple treatments by considering each treatment as a separate query.

- Finally, as illustrated with the PCG metric, the L2R framework allows optimizing rankings for custom metrics. This creates the opportunity to include profit-centric metrics into the modeling phase. Future research could for example look into integrating the Maximum Profit Uplift (MPU) metric, specifically created for UM [13], into the L2R framework with the aim of obtaining rankings that result in higher profits.

\section{REFERENCES}

[1] S. Wager and S. Athey, "Estimation and inference of heterogeneous treatment effects using random forests," Journal of the American Statistical Association, vol. 113, no. 523, pp. 1228-1242, 2018.

[2] K. Imai, M. Ratkovic et al., "Estimating treatment effect heterogeneity in randomized program evaluation," The Annals of Applied Statistics, vol. 7, no. 1, pp. 443-470, 2013.

[3] M. Qian and S. A. Murphy, "Performance guarantees for individualized treatment rules," Annals of statistics, vol. 39, no. 2, p. 1180, 2011.

[4] U. Shalit, F. D. Johansson, and D. Sontag, "Estimating individual treatment effect: generalization bounds and algorithms," in Proceedings of the 34th International Conference on Machine LearningVolume 70. JMLR. org, 2017, pp. 3076-3085.

[5] F. Devriendt, D. Moldovan, and W. Verbeke, "A literature survey and experimental evaluation of the state-of-the-art in uplift modeling: A stepping stone toward the development of prescriptive analytics," Big data, vol. 6, no. 1, pp. 13-41, 2018.

[6] S. Jaroszewicz and P. Rzepakowski, "Uplift modeling with survival data," in ACM SIGKDD Workshop on Health Informatics (HIKDD-14), New York City, 2014.

[7] R. M. Gubela, S. Lessmann, and S. Jaroszewicz, "Response transformation and profit decomposition for revenue uplift modeling," European Journal of Operational Research, vol. 283, no. 2, pp. 647 - 661, 2020. [Online]. Available: http://www.sciencedirect.com/science/article/pii/S0377221719309415

[8] E. Ascarza, "Retention futility: Targeting high-risk customers might be ineffective," Journal of Marketing Research, vol. 55, no. 1, pp. 80-98, 2018.

[9] T.-Y. Liu et al., "Learning to rank for information retrieval," Foundations and Trends( $\mathrm{R}$ in Information Retrieval, vol. 3, no. 3, pp. 225331, 2009.

[10] D. B. Rubin, "Causal inference using potential outcomes: Design, modeling, decisions," Journal of the American Statistical Association, vol. 100, no. 469, pp. 322-331, 2005.

[11] P. W. Holland, "Statistics and causal inference," Journal of the American statistical Association, vol. 81, no. 396, pp. 945-960, 1986.

[12] Diemert Eustache, Betlei Artem, C. Renaudin, and A. MassihReza, "A large scale benchmark for uplift modeling," in Proceedings of the AdKDD and TargetAd Workshop, KDD, London, United Kingdom, August, 20, 2018. ACM, 2018.

[13] F. Devriendt and W. Verbeke, "The case for prescriptive analytics: a novel maximum profit measure for evaluating and comparing customer churn prediction and uplift models," Vrije Universiteit Brussel, Faculteit Economische en Sociale Wetenschappen \& Solvay Business School, Belgium, WorkingPaper 12, 42018.

[14] L. Y.-T. Lai, "Influential marketing: a new direct marketing strategy addressing the existence of voluntary buyers," Ph.D. dissertation, School of Computing Science-Simon Fraser University, 2006. 
[15] M. Jaskowski and S. Jaroszewicz, "Uplift modeling for clinical trial data," in ICML Workshop on Clinical Data Analysis, 2012.

[16] V. S. Y. Lo, "The true lift model: A novel data mining approach to response modeling in database marketing," SIGKDD Explor. Newsl., vol. 4, no. 2, pp. 78-86, Dec. 2002. [Online]. Available: http://doi.acm.org/10.1145/772862.772872

[17] K. Kane, V. S. Lo, and J. Zheng, "Mining for the truly responsive customers and prospects using true-lift modeling: Comparison of new and existing methods," Journal of Marketing Analytics, vol. 2, no. 4, pp. 218-238, Dec 2014. [Online]. Available: https://doi.org/10.1057/jma.2014.18

[18] L. Breiman, J. Friedman, R. Olshen, and C. Stone, Classification and Regression Trees. New York, NY, U.S.A.: Chapman and Hall, 1984.

[19] G. V. Kass, "An exploratory technique for investigating large quantities of categorical data," Appl Stat, pp. 119-127, 1980.

[20] N. J. Radcliffe and P. D. Surry, "Real-world uplift modelling with significance-based uplift trees," White Paper TR-2011-1, Stochastic Solutions, 2011.

[21] P. Rzepakowski and S. Jaroszewicz, "Decision trees for uplift modeling with single and multiple treatments," Knowl Inf Syst, vol. 32, no. 2, pp. 303-327, 2012. [Online]. Available: http://dx.doi.org/10.1007/s10115-011-0434-0

[22] L. Guelman, M. Guillen, and A. M. Pérez-Marín, "Optimal personalized treatment rules for marketing interventions: A review of methods, a new proposal, and an insurance case study," Universitat de Barcelona, UB Riskcenter, Working Papers 2014-06, 2014. [Online]. Available: http://ideas.repec.org/p/bak/wpaper/201406.html

[23] L. Zaniewicz and S. Jaroszewicz, "Support vector machines for uplift modeling," in Data Mining Workshops (ICDMW), 2013 IEEE 13th International Conference on, 2013, pp. 131-138.

[24] F. Kuusisto, V. S. Costa, H. Nassif, E. Burnside, D. Page, and J. Shavlik, "Support vector machines for differential prediction," in Joint European Conference on Machine Learning and Knowledge Discovery in Databases. Springer, 2014, pp. 50-65.

[25] Ł. Zaniewicz and S. Jaroszewicz, "Lp-support vector machines for uplift modeling," Knowledge and Information Systems, vol. 53, no. 1, pp. 269-296, 2017.

[26] J. Yoon, J. Jordon, and M. van der Schaar, "Ganite: Estimation of individualized treatment effects using generative adversarial nets," in International Conference on Learning Representations, 2018.

[27] A. Betlei, E. Diemert, and M.-R. Amini, "Uplift prediction with dependent feature representation in imbalanced treatment and control conditions," in International Conference on Neural Information Processing. Springer, 2018, pp. 47-57.

[28] N. J. Radcliffe, "Using control groups to target on predicted lift: Building and assessing uplift models," Direct Market J Direct Market Assoc Anal Council, vol. 1, pp. 14-21, 2007.

[29] H. Nassif, F. Kuusisto, E. S. Burnside, and J. W. Shavlik, "Uplift modeling with roc: An srl case study." in ILP (Late Breaking Papers), 2013, pp. 40-45.

[30] P. Rzepakowski and S. Jaroszewicz, "Decision trees for uplift modeling," in Proceedings of the 2010 IEEE International Conference on Data Mining, ser. ICDM '10. Washington, DC, USA: IEEE Computer Society, 2010, pp. 441-450. [Online]. Available: http://dx.doi.org/10.1109/ICDM.2010.62

[31] — , "Uplift modeling in direct marketing," Journal of Telecommunications and Information Technology, pp. 43-50, 2012.

[32] M. Sołtys and S. Jaroszewicz, "Boosting algorithms for uplift modeling," arXiv preprint arXiv:1807.07909, 2018.

[33] M. Soltys, S. Jaroszewicz, and P. Rzepakowski, "Ensemble methods for uplift modeling," Data Min Knowl Discov, pp. 1-29, 2014. [Online]. Available: http://dx.doi.org/10.1007/s10618-0140383-9

[34] R. M. Gubela, S. Lessmann, J. Haupt, A. Baumann, T. Radmer, and F. Gebert, "Revenue uplift modeling," 2017.

[35] P. Gutierrez and J.-Y. Gerardy, "Causal inference and uplift modelling: A review of the literature," in Proceedings of The 3rd International Conference on Predictive Applications and APIs, ser. Proceedings of Machine Learning Research, C. Hardgrove, L. Dorard, K. Thompson, and F. Douetteau, Eds., vol. 67. Microsoft NERD, Boston, USA: PMLR, 11-12 Oct 2017, pp. 1-13. [Online]. Available: http://proceedings.mlr.press/v67/gutierrez17a.html

[36] L. Guelman, "Optimal personalized treatment learning models with insurance applications," 2015.
[37] C. Manning, P. Raghavan, and H. Schütze, "Introduction to information retrieval," Natural Language Engineering, vol. 16, no. 1, pp. 100-103, 2010

[38] C. J. Burges, "From ranknet to lambdarank to lambdamart: An overview," Tech. Rep. MSR-TR-2010-82, June 2010. [Online]. Available: https://www.microsoft.com/enus/research/publication/from-ranknet-to-lambdarank-tolambdamart-an-overview/

[39] Q. Wu, C. J. Burges, K. M. Svore, and J. Gao, “Adapting boosting for information retrieval measures," Information Retrieval, vol. 13, no. 3, pp. 254-270, 2010.

[40] Z. Cao, T. Qin, T.-Y. Liu, M.-F. Tsai, and H. Li, "Learning to rank: from pairwise approach to listwise approach," in Proceedings of the 24th International Conference on Machine learning, 2007, pp. 129-136.

[41] J. Guiver and E. Snelson, "Bayesian inference for plackett-luce ranking models," in Proceedings of the 26th International Conference on Machine Learning, 2009, pp. 377-384.

[42] K. Järvelin and J. Kekäläinen, "Cumulated gain-based evaluation of ir techniques," ACM Trans. Inf. Syst., vol. 20, no. 4, pp. 422-446, Oct. 2002. [Online]. Available: http://doi.acm.org/10.1145/582415.582418

[43] W. Chen, T. yan Liu, Y. Lan, Z. ming Ma, and H. Li, "Ranking measures and loss functions in learning to rank," in Advances in Neural Information Processing Systems 22, Y. Bengio, D. Schuurmans, J. D. Lafferty, C. K. I. Williams, and A. Culotta, Eds. Curran Associates, Inc., 2009, pp. 315-323. [Online]. Available: http://papers.nips.cc/paper/3708-ranking-measuresand-loss-functions-in-learning-to-rank.pdf

[44] B. McFee and G. R. Lanckriet, "Metric learning to rank," in Proceedings of the 27th International Conference on Machine Learning, 2010, pp. 775-782.

[45] C. Burges, T. Shaked, E. Renshaw, A. Lazier, M. Deeds, N. Hamilton, and G. Hullender, "Learning to rank using gradient descent," in Proceedings of the 22Nd International Conference on Machine Learning, ser. ICML '05. New York, NY, USA: ACM, 2005, pp. 89-96. [Online]. Available: http://doi.acm.org/10.1145/1102351.1102363

[46] W. B. Croft, D. Metzler, and T. Strohman, Search engines: Information retrieval in practice. Addison-Wesley Reading, 2010, vol. 520.

[47] T. Chen, T. He, M. Benesty, V. Khotilovich, Y. Tang, H. Cho, K. Chen, R. Mitchell, I. Cano, T. Zhou, M. Li, J. Xie, M. Lin, Y. Geng, and Y. Li, xgboost: Extreme Gradient Boosting, 2018, r package version 0.81.0.1. [Online]. Available: https://github.com/dmlc/xgboost

[48] J. Fürnkranz, E. Hüllermeier, and S. Vanderlooy, "Binary decomposition methods for multipartite ranking," in Joint European Conference on Machine Learning and Knowledge Discovery in Databases. Springer, 2009, pp. 359-374. 


\section{APPENDIX}

\section{Appendix A: AUUC for the Separate Relative Uplift Curve}

By substituting the definition of the Separate Relative Uplift Curve (Equation 4) into the AUUC definition for the separate setting (Equation 10), we obtain:

$$
\begin{aligned}
A U U C & \approx \sum_{p^{\prime}=1}^{100} V\left(p=\frac{p^{\prime}}{100}\right) \\
& \approx \sum_{p^{\prime}=1}^{100}\left(\frac{R\left(\mathcal{T}, p^{\prime}|\mathcal{T}| / 100\right)}{|\mathcal{T}|}-\frac{R\left(\mathcal{C}, p^{\prime}|\mathcal{C}| / 100\right)}{|\mathcal{C}|}\right) .
\end{aligned}
$$

Recall that $R(\mathcal{T}, k)$ and $R(\mathcal{C}, k)$ denote the number of treated and control responders among the top- $k$ ranked instances for the treatment and control groups, respectively. As these quantities are obtained by summations over the top- $k$ instances in the treatment and control groups separately, denoted by $k_{\mathcal{T}}=p|\mathcal{T}|$ and $k_{\mathcal{C}}=p|\mathcal{C}|$ for some percentage $p$, we can reformulate the above equation in terms of $k_{\mathcal{T}}, k_{\mathcal{C}}$ and the helper function $g(i)$ as defined in Equation 19, where $i$ represents a single instance, as follows:

$$
\begin{aligned}
A U U C & =\sum_{k_{\mathcal{T}}=1}^{|\mathcal{T}|} \sum_{i=1}^{k_{\mathcal{T}}} g(i)+\sum_{k_{\mathcal{C}}=1}^{|\mathcal{C}|} \sum_{i=1}^{k_{\mathcal{C}}} g(i) \\
& =\sum_{i=1}^{|\mathcal{T}|} \sum_{k_{\mathcal{T}}=i}^{|\mathcal{T}|} g(i)+\sum_{i=1}^{|\mathcal{C}|} \sum_{k_{\mathcal{C}}=i}^{|\mathcal{C}|} g(i) \\
& =\sum_{i=1}^{|\mathcal{T}|}(|\mathcal{T}|-i+1) g(i)+\sum_{i=1}^{|\mathcal{C}|}(|\mathcal{C}|-i+1) g(i) .
\end{aligned}
$$




\section{Summary of Changes: Learning to Rank for Uplift Modeling (TKDE-2020-04-0372)}

Dear Prof. Dr. Xuemin Lin,

We would like to thank the reviewers for the very helpful comments and remarks. We have substantially revised the paper in that we have streamlined the text and simplified the exposition of the uplift modeling evaluation measure framework. These two actions allowed us to shorten the paper. Further, we have also removed the appendices except for Appendix $A$ so as to focus on the most important findings. Below, we summarize the changes we made to the article in response to the reviewers' comments.

\section{Reviewer comments and author answers}

Dear Reviewers,

We would like to thank you for the time and effort you kindly invested in reviewing our manuscript. Your suggestions were constructive and helpful to us in improving the quality of our work.

Please find our answers to your comments and suggestions below. In the remainder of this document your comments have been formatted in bold and italic.

Once again, thank you for your remarks and suggestions. 
Reviewer \#1:

1. The present form of the paper is specific to uplift modeling. While having a strong interest in this field myself, I argue that uplift modeling is niche literature, which is about to be absorbed by the field of causal machine learning (CML). Parts of the paper are written like a classical uplift paper, which, in my opinion, is not suitable anymore because strong connections to the literature on treatment effects and causal inference are well-understood today and should be highlighted. The use of the do-operator and citing several relevant CML papers show that the authors are well familiar with the state-of-the-art. However, treatment effects and the conditional average treatment effect (CATE) in particular, which is equivalent to an uplift score, are never mentioned in the paper. To my knowledge, TDKE has not published previous work on uplift models. Thus, by focusing only on the uplift setting and marketing applications of uplift models, the authors leave an opportunity to highlight the generality of their work unexploited. I recommend i) revising the positioning of the paper and the review of related work such that connections to CML are indicated, and ii) revisiting the review of related work accordingly.

We have thoroughly reworked the introduction and Section 2 so as to clearly position the paper within the field of CML. Adjustments are also made to the other parts of the paper with this objective in mind. In the exposition of uplift modeling in Section 2, we now use the well-known Neyman-Rubin potential outcomes framework. However, in Sections 2.1 and 2.2 we intentionally keep the focus on the uplift modeling literature as the focus of this paper is to link the fields of uplift modeling (together with its evaluation measures) and L2R. In the related work section, we do, however, have included some extra CML references to recent work on ITE estimation.

2. Inconsistent use of evaluation measures is a crucial flaw of the uplift modeling literature and I am very appreciative of the paper introducing a unified framework of evaluation metrics (e.g., Table 2). This is an important contribution to the uplift modeling literature. Following my previous comment, I wonder whether measures like the uplift or qini curve might be unappreciated by the CML community in that the applicability of these measures might extend well beyond targeting marketing campaigns. With this in mind, I was somewhat disappointed that the authors focus exclusively on targeting settings. Given that the measures and their excellent synthesis are instrumental to the paper, I recommend that the authors attempt to discuss possible extensions of the use of these measures in other settings. The reason for this request is that I expect a corresponding discussion to make the paper more interesting for readers outside the field of uplift modeling.

We definitely agree that these measures do not (yet) seem to be adopted beyond the uplift modeling literature, although for sure they may have a broader use in settings where the ranking of instances is the prime objective. Although many such settings may be envisioned, however, in literature only a limited variety of cases can be retrieved. Additionally, note that these measures apply to a binary setting (both a binary treatment and binary outcome), limiting their impact and relevance within the field of CML. Nonetheless, we have found and added an example (personalized medicine) beyond the targeting marketing/retention campaigns.

3. I acknowledge that the empirical part (Section 4) is well-organized. Nonetheless, I found the part to be somewhat hard to follow at times. The authors could clarify the intention of a sub-experiment in a more approachable manner. For example, Section 4.3 starts with an explicit statement of the research questions to be tested, which is useful, whereas Section 4.2 does not and only hints at the goal of the test after elaborating on the simulation approach. The paper would benefit from a clear description of the steps one needs to take to use L2R for uplift modeling. This description does not have to be part of Section 4. It is clear that the chain of experiments in Section 4 is already the product of the authors' thinking what it takes to use L2R for uplift modeling. However, this thinking, one may call it a conceptual model, is never fully explicated in the paper. Instead, multiple parts of the paper including the introduction, the empirical part, and its discussion as well as the conclusions provide pieces of information. A flow chart of steps or something alike might be a solution but simply prose might be equally effective. Either way, I recommend that the authors identify a single, most suitable place in the paper in which the elaborate on the steps needed to use L2R for uplift modeling. They can then refer to this part when explaining the specific contributions of the paper and/or the experiments undertaken to implement the steps. 
We have included a new section (Section 3.3) in which we summarize the steps needed to use L2R for uplift modeling. We also use this section (refer to the different steps) to explain the goals of the different experiments in the reworked introduction of Section 4.

4. Arguably this point is related to be the previous one as also concerning the empirical part. The exposition is rather descriptive at times. I understand that you leave a discussion and interpretation of results for Section 5. However, I suggest you re-read the paper and decide whether there is room for streamlining the exposition and focusing it on the most important findings, which are not immediately available in the tables or figures.

We have refined the exposition and discussion of the results in that we have streamlined the text and tried to focus on the most important findings. In this regard, we have also removed the appendices except for Appendix A.

5. The experiments concerning research question 1 involve defining three scenarios, which differ in terms of the ratio of treatment to control group observations. At this point, I was wondering whether an outcome modification such as inverse propensity weighting or the doubly robust approach would not overcome any imbalance in the number of group members and facilitate the use of an uplift measure without worrying about stability. I do not associate any specific recommendation with this point. The authors may reflect connections to modified outcome methods in the paper if they feel this would add value to the section, or simply ignore the comment.

This is an important point and a true concern with respect to the practical application of CML. For quite some time, in our lab we have been discussing about the impact of imbalanced data sets (both in terms of treatment/control distribution, as well as class distribution) on both the stability and performance of uplift models, and carrying out some exploratory experiments. It is a difficult issue to tackle and discuss, though, and we thought it to be better not to touch upon it in this paper, since it is not the core focus of the paper and the best we could do is to indicate it as a prime topic for future research and provide some initial thoughts and directions to explore. Hence, if permitted, we would prefer not to discuss upon it. By providing this comment, however, the reviewer strengthens our believe that further research on this topic may be of interest to and hence appreciated by the community.

6. Since you stress notation and precision, which is missing in prior work on uplift/Qini curves, I was thinking about equation (5) and (6). If using the Iverson bracket, these equations appear to translate into calculating a sum over products of the number of responders and treated observations (or untreated observations in equation 6). This is not what you mean. Maybe revisit whether the notation with two brackets actually is the right way to express the counting that you have in mind.

We have simplified the exposition of the uplift modeling evaluation measure framework in the revised version of the manuscript. In the revised version of the manuscript, the Iverson bracket is not used anymore.

\section{Minor comments}

a. The reference to Table 2 on page 8 (bottom) in Section 4.1.1 seems wrong. Should this be a reference to Table 5?

b. Check the numbering of the sub-sub sections in Section 4.2. Shouldn't it be 4.2.1 instead of 4.2.0.1.?

Corrections were made to the manuscript to address all the comments above. 
Reviewer \#2:

1. One remark is in place here: from the modeling point of view Sep-Abs Qini curve is identical to the SepRel Uplift Curve as one can be obtained from another by dividing by IT/ which is a constant. The Sep-Abs Qini curve is thus a relative not Absolute measure. The authors do write about the equivalence but only on page 11. I believe it should be stated much earlier and reflected in Table 2 and related text. This also affects the experiments in Section 4.2 where curves for some methods are simply rescaled curves for other methods. This should be clearly indicated.

In the revised version of the manuscript, we discuss the equivalence between the two curves already in Section 2.2. In Section 4.2, we now also explicitly state that the curves in Figure 3a are rescaled versions of the ones depicted in Figure 2a. However, we do not explicitly reflect the equivalence in Table 2, as we categorize the Sep-Abs Qini curve as an absolute measure since we use the term 'absolute' to refer to the way the cumulative incremental gains are expressed (also see the caption of Table 2), which is in abolute terms for the Sep-Abs Qini curve.

2. Typos:

a. page 4, line 24: "It aims to directly optimizes"

b. page 10 line 22: "... in joint setting have this." It is unclear what

c. Figure 4: different dataset names (Insurance, Clothing) are used in the Figure and in the tables (Information, Hillstrom). Same in Fig 7

Corrections were made to the manuscript to address all the comments above. 
Reviewer \#3:

1. Although the topic is interesting and the paper is easy to follow, I found it quite lengthy, partly a bit wordy and tough to read.

We have substantially revised the paper in that we have streamlined the text and simplified the exposition of the uplift modeling evaluation measure framework. These two actions allowed us to shorten the paper. Further, we have also removed the appendices except for Appendix A so as to focus on the most important findings.

2. There is essentially no theory, only experiments on three data sets. As with all purely empirical papers, one should therefore be cautious with conclusions. In particular, on should also consider the specific choice of the methods and other decisions regarding the experimental setup. That said, I find it difficult to make concrete suggestions for improvement. Perhaps one could try to improve the presentation a bit, making it more concise and to the point (there are also a number of typos and small grammatical mistakes).

We have refined the exposition and discussion of the results and we have paid explicit attention not to overstate the significance of the findings. In the conclusion, we also indicate the need for further research (e.g., on alternative relevance value sets) and more extensive empirical results.

3. As for the learning-to-rank methods, I think the presentation could be a bit broader, both regarding methods and performance metrics. For examples, even if such methods are not necessarily used and included in the experiments, I could imagine that model-based methods might be an interesting alternative to (non-parametric) approaches such as LambdaMART. In particular, the Plackett-Luce model has become quite popular in the LTA field [1,2]. In this (probabilistic) model, each alternative has a latent "skill" parameter, which might be interpreted as a degree of susceptibility in the context of uplift modeling (interpretability is clearly an appealing property). Since the model is probabilistic, it can be learned using maximum likelihood inference.

[1] John Guiver, Edward Snelson. Bayesian inference for Plackett-Luce ranking models. ICML 2009.

[2] https://arxiv.org/abs/1909.06722

We agree that the presentation of the learning to rank methods is rather focused on the LambdaMART technique. Therefore, in Section 3, we now mention the existence of alternative methods and have included some references to model-based methods. However, as the focus of this paper is to explore the link between learning to rank and uplift modeling, we do not discuss these methods in detail. A broader exploration of learning to rank methods for uplift modeling is however identified as a prime topic for further research (in Sections 3 and 6).

4. As for the performance metrics, the authors essentially focus on metrics that are commonly used in information retrieval, i.e., measures that are based on relevance degrees of the items. However, the relevance degrees assigned to the four types of customers (treated responders non-responders, control responders and non-responders) are of course a bit arbitrary. Alternatively, the ranking problem could be tackled as a problem of multi-partite ranking [3,4], which is an extension of bipartite ranking. In multi-partite ranking, the population is divided into different groups, and the goal is to establish a ranking that is coherent with these groups. In uplift modeling, for example the goal would be to rank treated responders higher than non-responders higher than control non-responders higher than control responders. As performance measures, the C-index (a generalization of AUC) is commonly used in multipartite ranking. It essentially counts the number of violations in the ranking, i.e., the number of pairs $(a, b)$ such that $a$ is ranked higher than $b$, although the opposite should be the case.

[3] J. Fürnkranz, E. Hüllermeier and S. Vanderlooy. Binary Decomposition Methods for Multipartite Ranking. ECML/PKDD 2009.

[4] A. Fallah Tehrani, W. Cheng, E. Hüllermeier. Preference Learning using the Choquet Integral: The Case of Multipartite Ranking. IEEE Transactions on Fuzzy Systems, 20(6):1102-1113, 2012.

We agree that it is an interesting idea to cast the problem as a multipartite ranking problem. This is also indicated in Sections 5 and 6 by including it as a possible direction for future research. However, as we 
focus on the LambdaMART technique as learning to rank method in this paper, we have chosen to keep the discussion on the learning to rank metrics limited to the metrics that can be used by LambdaMART to optimize rankings. 